\title{
Wang tiling for particle heterogeneous materials: Algorithms for generation of tiles/cubes via molecular dynamics
}

\author{
D. Šedlbauer ${ }^{a, *}$, M. Lepš $\check{~}^{a}$ \\ ${ }^{a}$ Department of Mechanics, Faculty of Civil Engineering, CTU in Prague, Thákurova 7/2077, 16629 Prague, Czech Republic \\ Received 8 March 2019; accepted 25 June 2019
}

\begin{abstract}
This paper aims at a reduction of periodicity artefacts during a generation of random heterogeneous material models. The traditional concept of the Periodic Unit Cell is compared with a novel approach of the stochastic Wang tiling. Since modelled structures consist of hard circular/spherical particles in a matrix, the algorithm for placement of inclusions is based on the modified molecular dynamics. We introduce two types of Wang tile boundary conditions to decrease periodicity artefacts. Tested samples for 2D applications form sets of both monodisperse and polydisperse microstructures. The overall volume fractions of these samples are approximately $0.2,0.4$, and 0.6 , respectively. The generated sets are analysed both visually and statistically via a two-point probability function. An extension of the stochastic Wang tiling enables to create 3D structures, as well. Therefore, artificial periodicity is also investigated on a 3D sample consisting of spherical particles of identical radii distributed in a continuous phase.
\end{abstract}

(c) 2019 University of West Bohemia. All rights reserved.

Keywords: random heterogeneous material, Wang tiling, Periodic Unit Cell, molecular dynamics

\section{Introduction}

The most of real-world materials can be considered as heterogeneous (composed of different materials or the same material in different states [29]) at least on the micro or nanoscale level. Here, the structure can be characterised statistically based on information about the geometry and the location of individual components. The homogenisation approaches together with the concepts of the Periodic Unit Cell (PUC) or the Statistically Equivalent Periodic Unit Cell (SEPUC) [15] or [28], and the Representative Volume Element (RVE) [10, 20, 24] or [1], are applied to determine the effective properties at higher microstructure levels.

The above methods can be used for both tasks of reconstruction and compression of the random heterogeneous materials. Unfortunately, we do not avoid the loss of information about the randomness of the domain, thereby characteristics or properties of the material samples are distorted. The modelling of the random heterogeneous microstructures can be understood as a creation of 2D surfaces or 3D spaces of finite dimensions. Similar tasks have been successfully solved via Wang tiling (WT) $[30,31]$ in various fields of science. Let us mention just a few of them to indicate the widespread application of WT principles. The generalized main advantage for such a tiling is the ability to create infinite random heterogeneous domains via a finite set of basic building blocks - Wang tiles.

Ares Lagae in his thesis deals with Wang and corner tiling for solving complex signals [16]. Cohen and his colleagues define basic principles of stochastic tiling as an alternative for the

${ }^{*}$ Corresponding author. Tel.: +420 224354 495, e-mail: david.sedlbauer@fsv.cvut.cz. https://doi.org/10.24132/acm.2019.537 
strictly aperiodic version of the WT [3]. Both of these works belong originally to computer graphics and are utilised in the following sections of this work. The WT can be found in medicine and drug industry, as well. The work introduced by Winfree and his co-workers utilise WT for the design of DNA [32]. History of DNA nanotechnology including tiling applications can be found in the contribution of Seeman and Sleimann [25]. In general biology, Klein et al. presented WT with a fast population of membrane lipids [14]. Over the last few years, principles of the WT concept have been taken over and incorporated into material engineering, where they extend the SEPUC concept [21,22] or [7].

Wang tiling in material engineering with emphasis on particle domains makes it possible to reduce the number of particles and, thus, computational demands, while keeping or increasing the degree of heterogeneity of (re)constructed samples. This statement is valid for both domains created without portioning to the grid of tiles and those with tiles of strictly periodic conditions [21]. With the application of statistical descriptors, we are able to produce models of required properties without primer creation and analysis of in-situ samples. These principles reduce overall costs, while dealing with both new artificial and real-world material structures.

The present paper aims at modelling of random heterogeneous material samples composed of discrete hard circular or spherical particles in a matrix. Illustrative examples are composites, where the basic domain is reinforced with fibres (reinforced concrete, textile belts) and/or where a rigid structure forms the matrix and pores represent particles (metal foams, plastic), Fig. 1. The main goal of the presented research is to create and compare similar domains based on the traditional PUC concept and a new Wang tiling method via the molecular dynamics. The work is organised as follows: the second section introduces general Wang tiling and specific Stochastic Wang tiling methods, the third section describes algorithms for a generation of tiling sets. Differences in samples composed of both the PUC and the Wang tiling processes are presented and discussed on comparative types of artificial material domains in the fourth section. The last paragraphs summarise the paper and outline possibilities for further work.
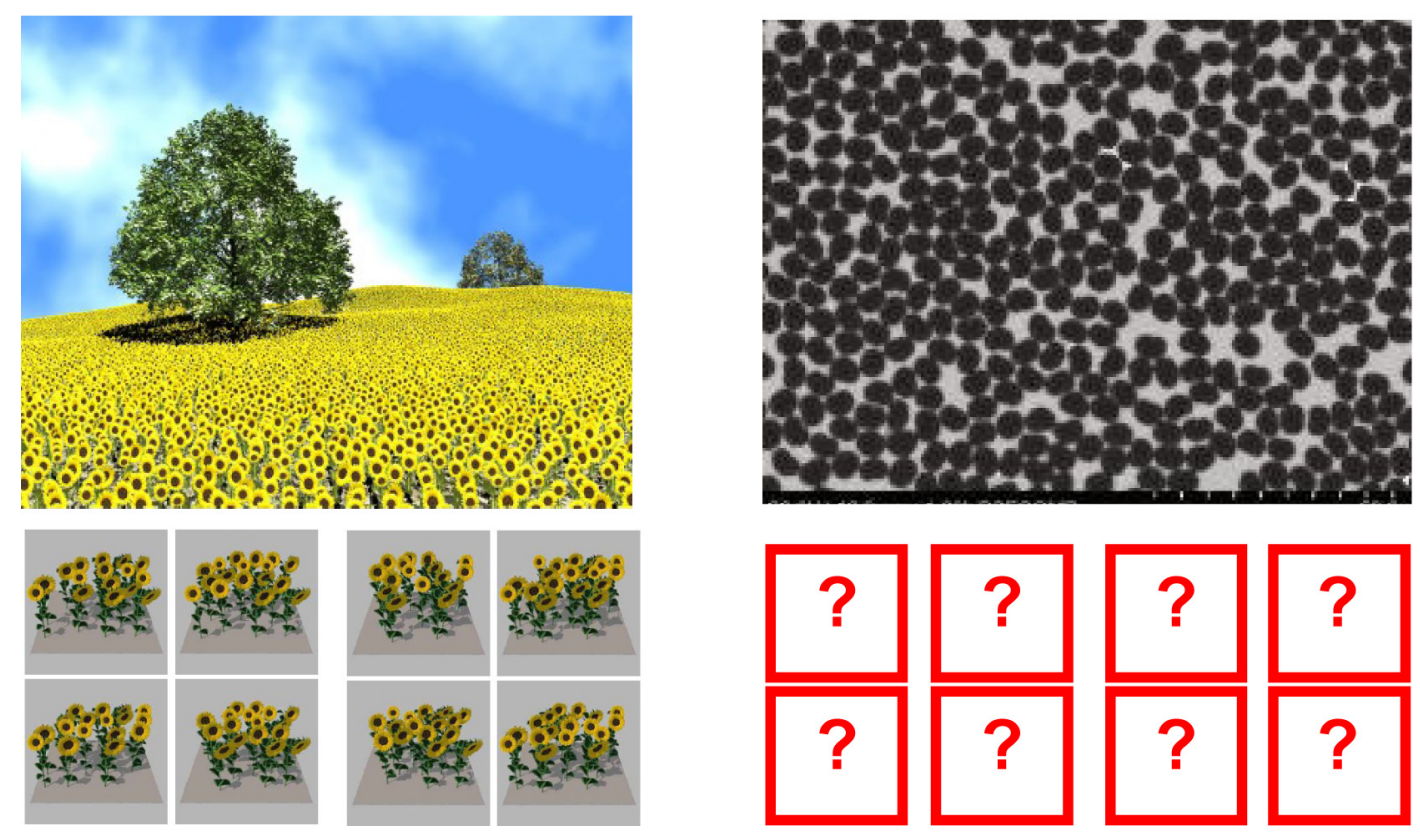

Fig. 1. Wang tiling applications: (left) set for computer graphics [3], (right) microstructure and free slots symbolising the main contribution issue - generation of Wang tile sets 


\section{Stochastic Wang tiling}

\subsection{Wang tiling}

The basis of the term Wang tiling goes back to the sixties of the twentieth century, when the mathematician Hao Wang introduced a set of tiles as a tool for the study of the decision problem [30,31]. Every single tile in a set was formed by a square with colours (codes) on each edge. During the tiling process, tiles were not permitted to rotate or reflect. The question was to find an effective procedure, which can decide whether copies of squares from the finite set are able to cover a whole plane (infinite plane) with restriction of adjoining edges - colours. This generalised game of dominos leads to the finding of periodically repeated areas. Through the years researches proved that there are sets of tiles that make it possible to cover a plate even without periodic repetition of a certain group of tiles [2]. The last two smallest sets, to the best knowledge of the author, are shown in Fig. 2. The first set consists of thirteen individuals with five different colours on edges [5]. The second set includes eleven tiles over only four codes [11]. Worth noting are the same codes on both vertical and horizontal edges in comparison with the Stochastic Wang tiling.
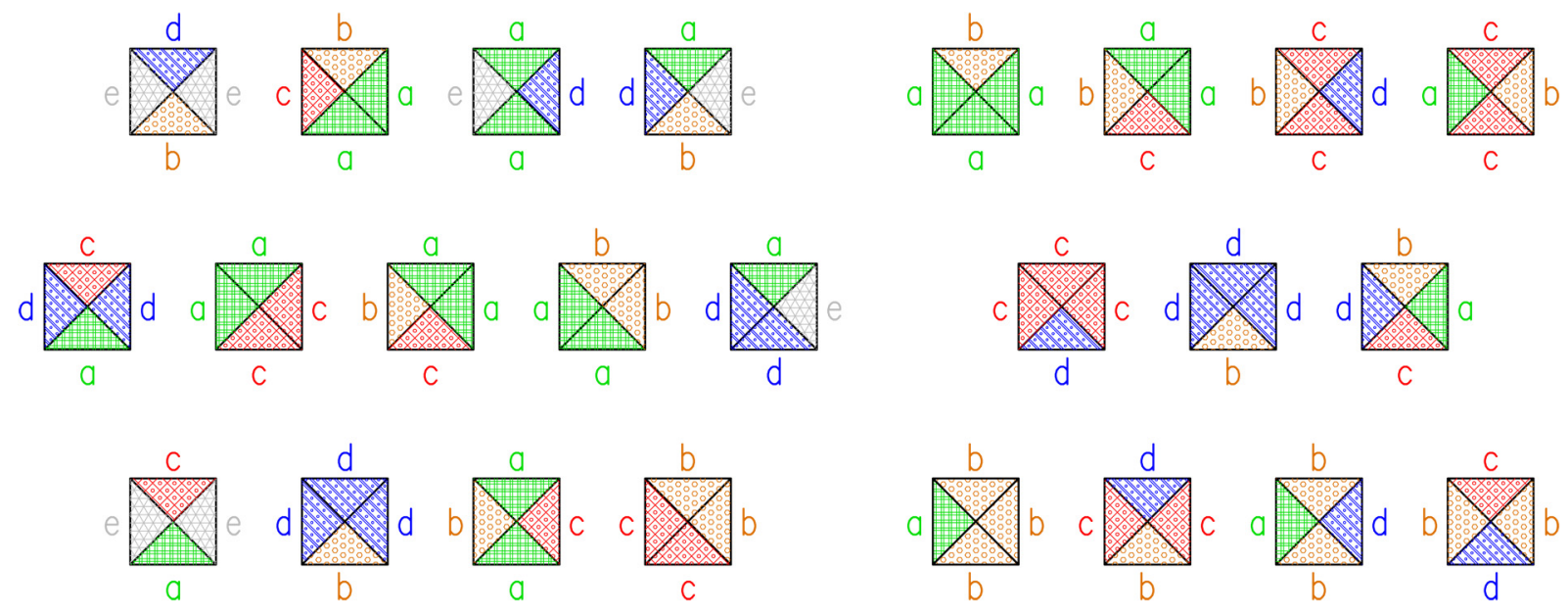

Fig. 2. Minimal sets for aperiodic tiling: (left) 13 tiles over 5 codes [5], (right) 11 tiles over 4 codes [11]

\subsection{Stochastic tiling}

Applications in material engineering do not require models with strictly aperiodic microstructure. Real heterogeneous material domains suffer from periodic artefacts, as well, representing, e.g., clusters of pores or, on the other hand, regularly arranged reinforced fibres. Thus, in this paper, an aperiodic tiling is replaced by the stochastic CSHD algorithm [3] for the creation of a tiling lattice.

Tiles from a basic tiling set in two dimensions are placed into the lattice column by column and row by row. In a general position of the process, the system has to propose at least two individuals for the next step in order to be called stochastic. These requirements together with the number of codes on an appropriate edge define a minimal number of tiles in the set. Considering $n_{x}$ and $n_{z}$ as numbers of different colours on vertical and horizontal edges respectively, the number of tiles in full or complete set is $n_{x}^{2} n_{z}^{2}$, whereas the minimal set forms $2 n_{x} n_{z}$ tiles, see Fig. 3. Extension to the 3D system is straightforward, Fig. 4. 


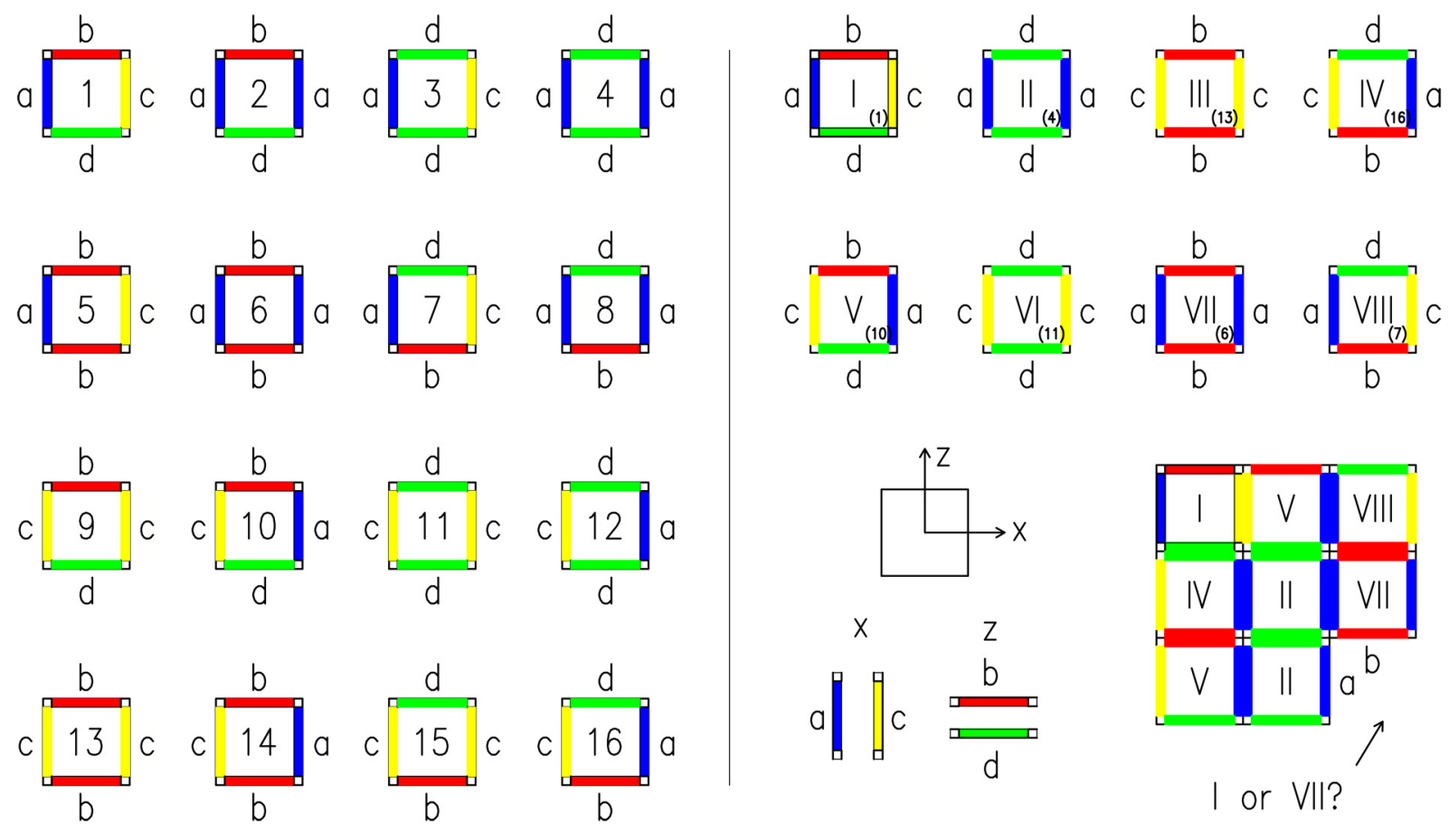

Fig. 3. Stochastic Wang tiling for 2-2 codes 2D: (left) full set, (right) minimal set with the algorithm of tiling
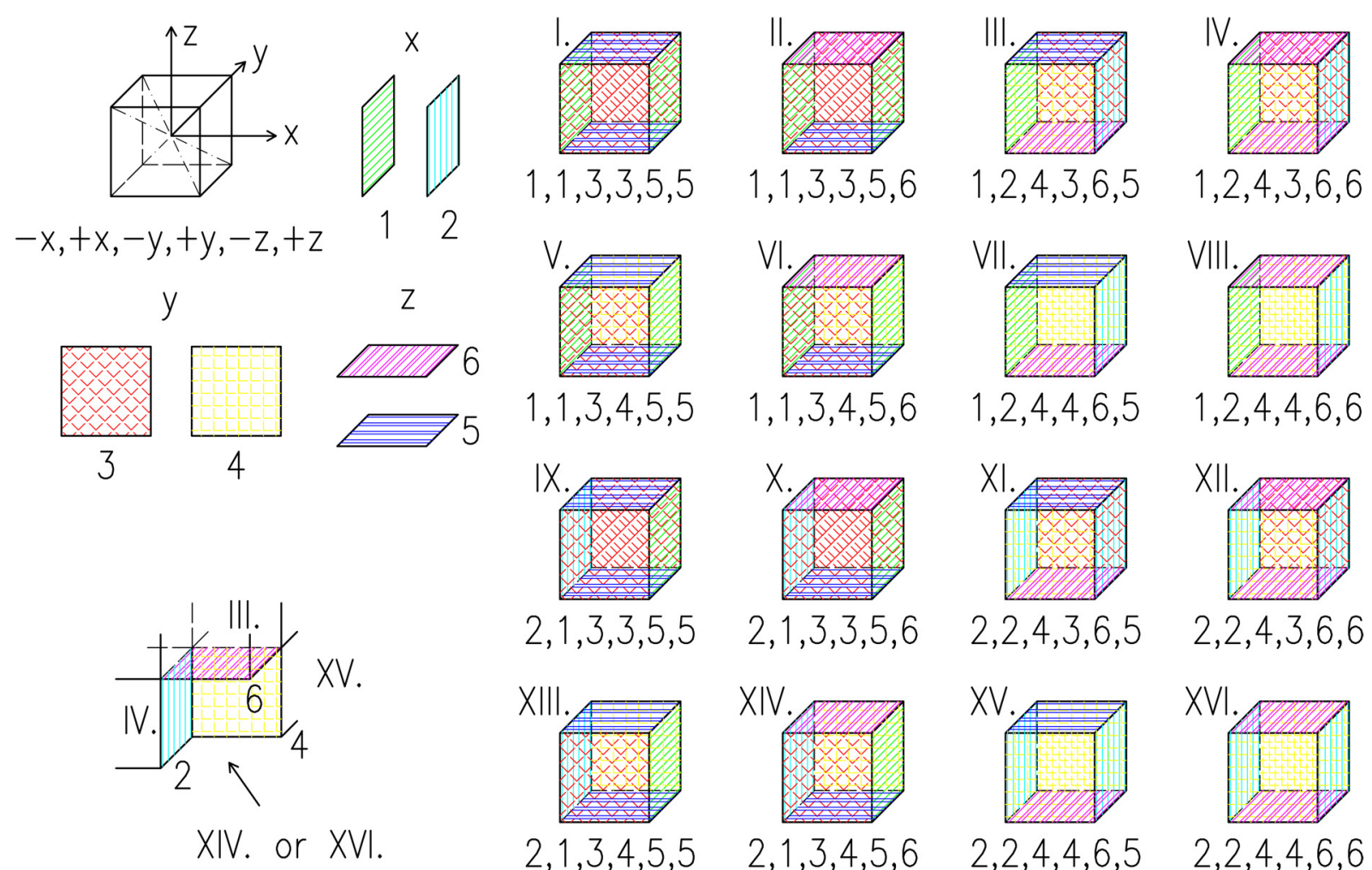

$1,2,4,4,6,5$

$1,2,4,4,6,6$
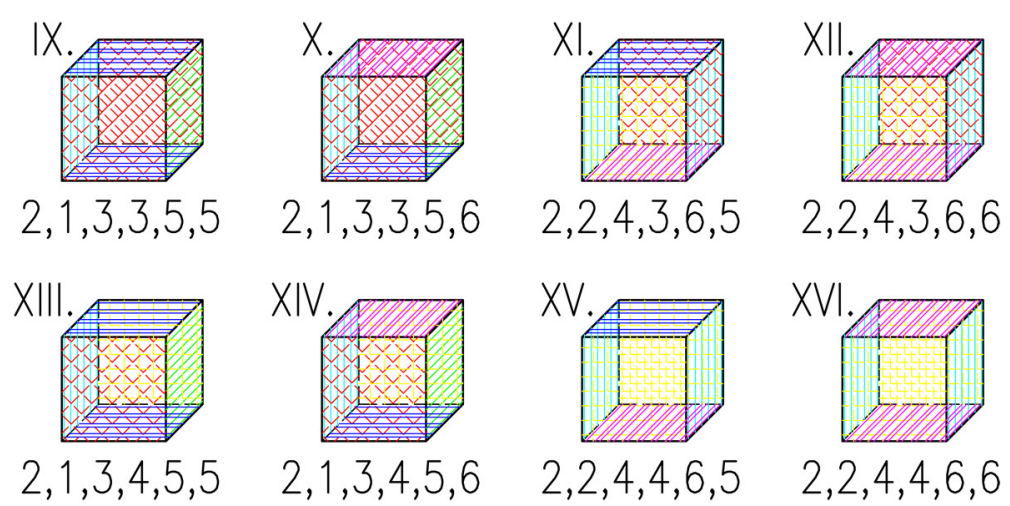

Fig. 4. Stochastic Wang tiling 3D for 2-2-2 codes: minimal set with the algorithm of tiling 


\section{Generation of Wang tiles}

\subsection{Molecular dynamics}

The investigated models of artificial material domains consist of impenetrable hard discs in a matrix. All of the algorithms for the generation of Wang tiles with boundary conditions utilise molecular dynamics, namely the modified Lubachevsky-Stillinger approach [18, 19]. Here hard particles represent molecules, whose motion and actual position manage initial random positions and velocity vectors instead of mutual attractive and repulsive forces. The final particle positions are based on a number of algorithm time steps in comparison with the traditional approach of optimal energy state.

At the beginning of the algorithm, particle centres are semi-randomly thrown into tiles. The term semi-randomly can be expressed with two steps. The first one requires knowledge of the prescribed particle volume fraction and the area ratio of parts into which the tile is divided, see the detailed description in the following sections. Within the second step, particle centres are randomly arranged in the appropriate tile parts. Concurrently with the process of placement, the particle centres are assigned random velocity vectors with maximal value equal to the tenth of the tile edge size. The whole system is released right after the setting of initial movement control parameters. During the algorithm particles grow, collide with each other and bounce off the tile walls in accordance with principles of elastic collisions. In the case, where a material model has no required properties, the stopping criterion for the motion is represented by the reaching of the final radii of the particles [27].

There are three types of parameters managing the process: fixed inputs - requirements given by the nature of Wang tiling, variable inputs - based on the type of modelled microstructure and variables - user defined. The physical size of tiles, member of the group with fixed inputs, is set to be eight times greater than the highest final particle radius. Such setting, on one hand, provides tiles small enough for savings of computer demands, on the other hand, it enables particle movement with the guaranty of particle non-overlapping. It must be noted that volume fractions of tested artificial material domains are below maximal dense packing schemes [9] as well as maximal random jammed states [6]. The tile size also defines a range for particle velocities; the maximal value enables a particle centre to cross a tile in both horizontal and vertical direction within ten time steps.

The variable inputs are the particle volume fractions and the required particle radii. These parameters define, in the simplest case of equivalent tile volume fractions, the number of particles in each tile. The most time-consuming part during the process lies in the total number of collisions, which depends just on the number of hard discs, the norm of velocity vectors, and the total number of time steps. While the first and the second character are user-independent, the last one is the only member of the user-defined group of parameters. The lower the number of time-steps, the more the particle motion is restricted. If there is a lower number of time-steps $(<100)$, the particles get stuck nearby their initial position. Thus, this configuration forbids posterior utilisation of optimisation techniques for achieving better compliance with reference media or prescribed material properties.

\subsection{Boundary conditions - Periodic Unit Cell (PUC)}

The following sections enrich the general description of the molecular dynamic system with specific boundary conditions of an appropriate type of tiles. The first one is a traditional concept of the PUC. Let us consider a plane problem. If any particle crosses any of the vertical or horizontal tile edges, the copy of the same particle appears on the opposite side. The particle 
movement continues until the particle centre reaches the edge and the rebound or any other inclusion forces the original particle to change the velocity vector back to the tile. A similar algorithm works for three-dimensional problems, as well, only the edges are replaced with walls of the cube. Note that a minimal set of Wang tiles includes tiles with the same codes on opposite sides, which can be designated as PUCs.

\subsection{Boundary conditions - Volume Walls (VW)}

The situation with boundary conditions for molecular dynamics gets difficult considering a material with hard particles together with the Wang tiling approach. When the particle tends to leave the mother tile through the edge with a certain code, it has to be copied to every edge designated with the same code on any other tile in order to meet the requirements of the stochastic tiling. Such permission leads to unwanted increasing of overall volume fractions. Therefore, we present a concept with Volume Walls, where the volume fraction of the tile set remains the same during the whole process.

At the beginning of the generation, before particle centres are thrown into tiles, each tile is divided into central and border parts of a certain volume - Volume Wall [27]. The width of the VW is equal to the diameter of the largest particle in a model. One half of the VW is inside the tile, the other is outside and physically belongs to the neighbouring tile in a final domain. The number of particles within the border and central parts usually follows the ratio of its areas and prescribed volume fractions. The border parts have the same areas. Therefore, the number of particles within these parts should be the same, no matter of the code, to avoid unwanted cluster artefacts in the final microstructure. Even though there are rigid barriers between the tile parts, central particles can collide with the border ones. The rebound occurs only when the particle centre reaches the barrier, Fig. 5.

Note the corner parts, where particle centres never get to. This modification prevents copying of particles to the tile, which has no common boundary part with the master one. If there is no dead space, a particle leaving tile through the corner finds its copies all over the set. The aforementioned state is called a corner problem, which might be solved in a general case with
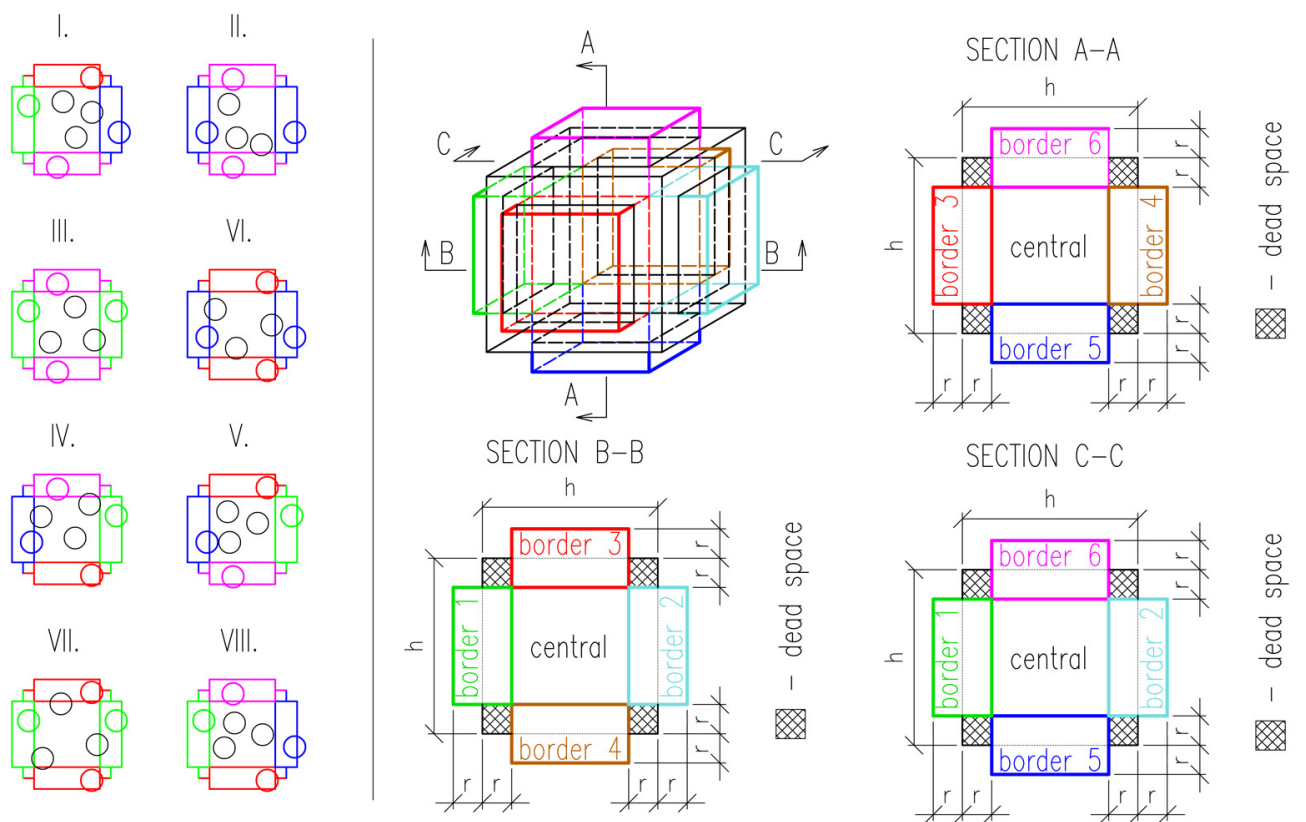

Fig. 5. Wang tiling with volume walls (VW): (left) 2D set, (right) division of one 3D tile into appropriate parts 
corner tiles [17]. Unfortunately, corner tiling does not fit to our case, where the tile set is generated via molecular dynamics. The nature of the algorithm only moves the problem to the middle of the tile edges [26].

\subsection{Boundary conditions - Adaptive walls (AW)}

The last proposed type of boundary conditions aims at reducing the disadvantages of the first two approaches. Random heterogeneous material domains generated with the concept of the PUCs consist of copies of just one tile, which brings a great number of unwanted periodicities. The Wang tiling method with Volume Walls significantly reduces the periodicity of the domain due to the stochastic tiling based on a set of different tiles. Notwithstanding this success, still, parts of tiles corresponding to the number of different codes on edges cause artificial periodicity artefacts. These artefacts get more significant with a higher volume fraction of the domain. For detailed comparison, see Section 4.

The main task for the improvement of tile sets generated via molecular dynamics is how to keep the compatibility of tiles without the repetition of tile parts. One of the solutions that satisfies this condition is the one that prevents any particle transition across the tile edge. Such system leads to material domains composed of independent blocks forming a lattice of vertical and horizontal lines without any particle. Contrary, a method similar to the puzzle system seems promising.

Let us consider one tile - a master one - where a moving particle tends to abandon the inner tile space. When the particle crosses a certain edge, all edges designated with the same code on the other tiles - slave tiles - adapt to the particle surface. This approach is called Adaptive Walls, Fig. 6. Even though the walls deform, the overall space for particle motions remains unchanged. Moreover, adapted edges take over dynamic parameters of the leaving particle. In other words, if any particle from any slave or master tile collides with the adapted part of the wall, the particle in the master tile changes its velocity vector like there is a direct collision of two particles. The reader is referred to [26] for a detailed description of the Wang tiles algorithm with Adaptive Walls. The extension to the 3D space is straightforward. The modification of edges is transferred to the walls.

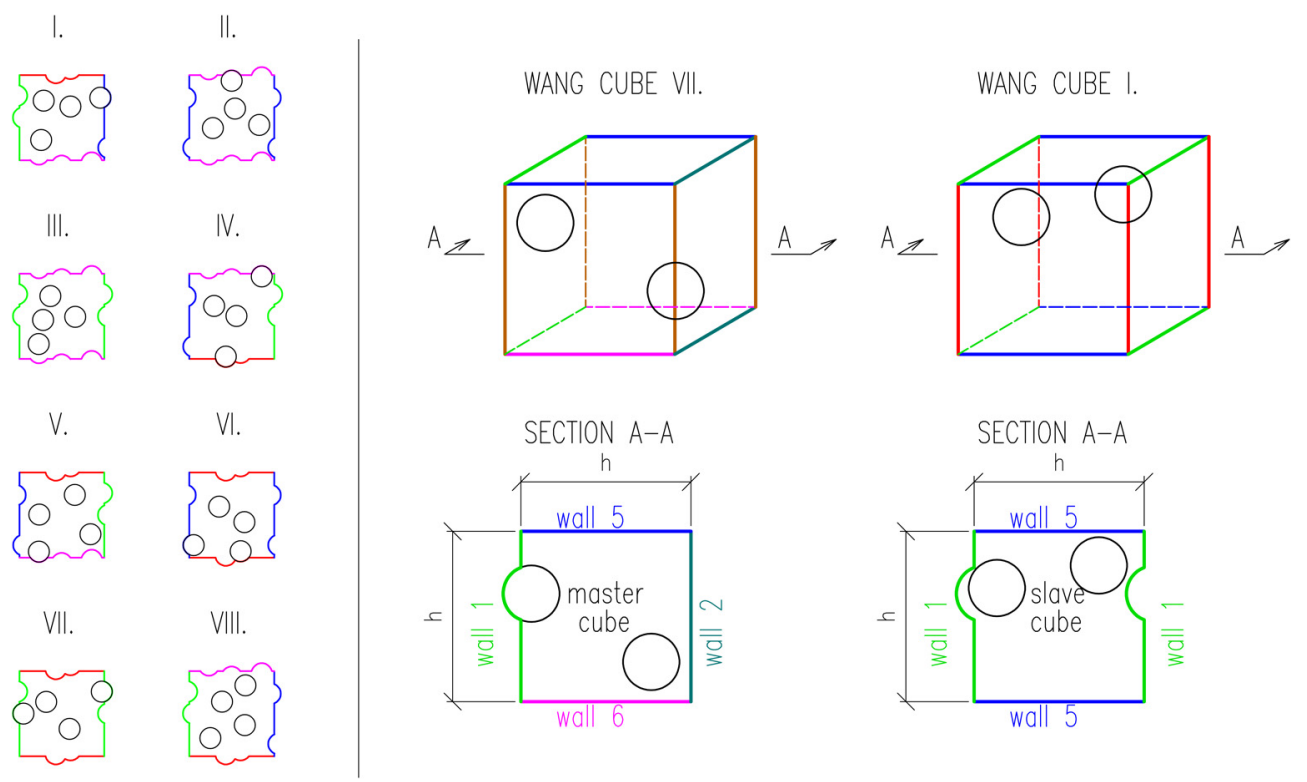

Fig. 6. Wang tiling with adaptive walls (AW): (left) 2D set, (right) visualization of AW algorithm in 3D (master cube + one slave cube) 


\section{Simulations and results}

This section aims at the comparison of the Wang tiling process with the concept of the PUC. We show the contrast in both visual and statistical ways using a lower order descriptor, namely the two-point probability function $[8,29]$. It has to be noted that structures are not optimised from a statistical point of view; the focus is aimed at a reduction of a significant accompanying phenomenon - secondary peaks - by applying different boundary conditions.

\subsection{Monodisperse 2D sets}

Every single example consists of four randomly generated samples: a single sample considered as the original one with required properties and three tilings assembled by tiles with the PUC, the VW, and the AW boundary conditions. The mesh of eight by eight tiles represents every tiled microstructure. The sequence of tiles is similar for each tiling within one comparable example. All tiles from the basic set in Wang tiling samples have the same number in a final domain. In general case of different tile volume fractions, the mentioned settings eliminate discrepancies in the final sample volume fractions. Only one group of samples for each example are shown for the purposes of visual comparison.

The first artificially modelled microstructures consist of equal sized hard discs of radius 15 pixels in a continuous phase. We tested domains of three different particle volume fractions: $0.2,0.4$, and 0.6 . The tile edge size is 100 pixels, in accordance with dynamic algorithm definition from the previous section. The numbers of particles in samples and/or tiles are summarised in Table 1. While the number of particles in the PUCs and Wang tiles with the AW proportionally corresponds to the overall volume fractions, the situation on tiles with the VW differs. If we consider two adjancent tiles with certain code/colour on edges, in the system with the VW particles in common border parts completely overlap. Therefore, the real number of particles per the VW tile equals to the sum of inclusions in the central part and the half of inclusions in all four border parts. Fig. 7 shows all types of microstructure generations for three modelled states.

Table 1. Specification of artificial monodisperse 2D samples

\begin{tabular}{|c|c|c|c|c|}
\hline $\begin{array}{c}\text { Volume } \\
\text { fraction app. }\end{array}$ & Method & $\begin{array}{c}\text { Particles } \\
\text { per tile }\end{array}$ & $\begin{array}{c}\text { Total number } \\
\text { of tiles }\end{array}$ & $\begin{array}{c}\text { Total number } \\
\text { of particles }\end{array}$ \\
\hline \multirow{4}{*}{0.2} & sample & - & - & 256 \\
\cline { 2 - 5 } & PUC & 4 & 64 & 256 \\
\cline { 2 - 5 } & VW & 5 & 64 & 256 \\
\cline { 2 - 5 } & AW & 4 & 64 & 256 \\
\hline \multirow{4}{*}{0.4} & sample & - & - & 512 \\
\cline { 2 - 5 } & PUC & 8 & 64 & 512 \\
\cline { 2 - 5 } & VW & 11 & 64 & 512 \\
\cline { 2 - 5 } & AW & 4 & 64 & 512 \\
\hline \multirow{5}{*}{0.6} & sample & - & - & 768 \\
\cline { 2 - 5 } & PUC & 12 & 64 & 768 \\
\cline { 2 - 5 } & VW & 17 & 64 & 768 \\
\cline { 2 - 5 } & AW & 12 & 64 & 768 \\
\hline
\end{tabular}




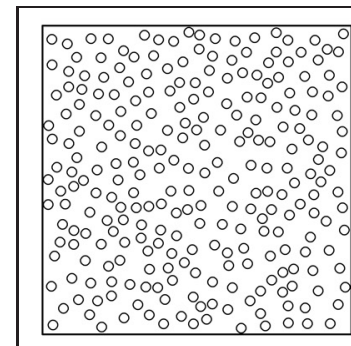

artificial sample

00000000000000000

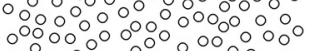

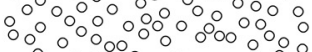
$0.00000000000 \%$

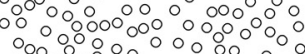

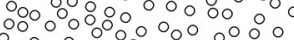

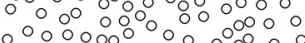

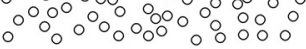

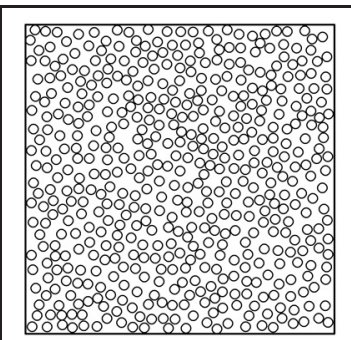

artificial sample

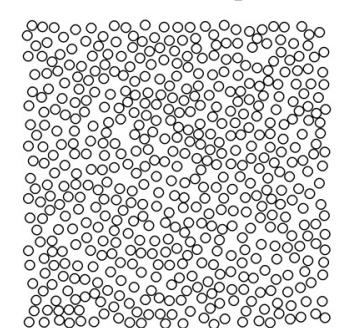
\begin{tabular}{l} 
8. \\
8080080000000000000000000000000 \\
\hline
\end{tabular}

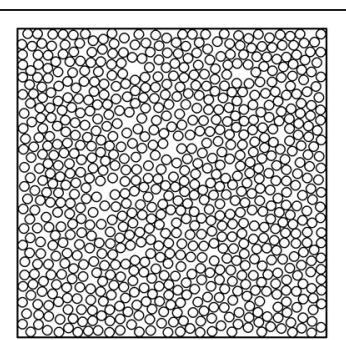
artificial sample

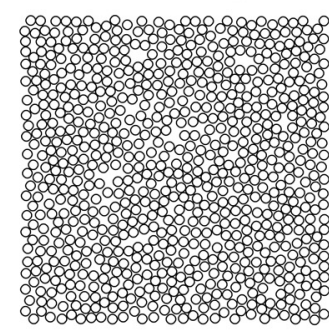

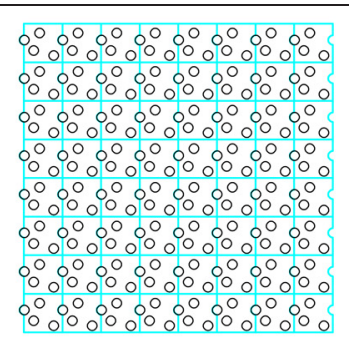

PUC; 4

$\because 00000000000000$ 0000000000000000 0000000000000000 00000000000000 00000000000000000 0000000000000000 0000000000000 00000000000000000

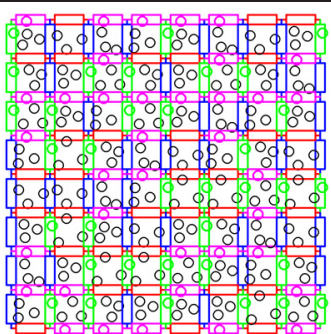

Wang tiling - VW; $\mathbf{n}$

$\therefore 000: 00000000000$ 0000000000000008

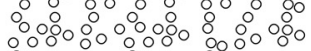
$\circ 00000800000000$ $0,000.000000000000000$

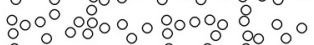

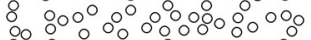
$\circ 0000000000000000$

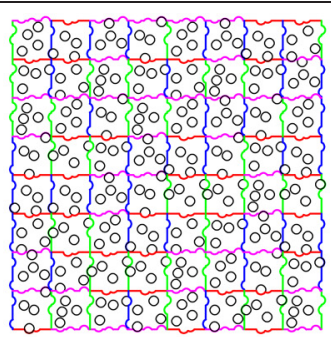

Wang tiling - AW; 44

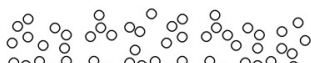

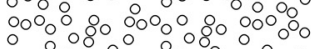

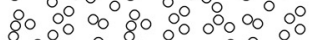
0000000.000000000 $\therefore \circ .0000008008000$ $\circ .00000 .00000000$

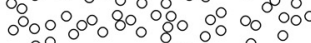

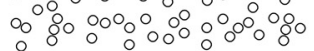
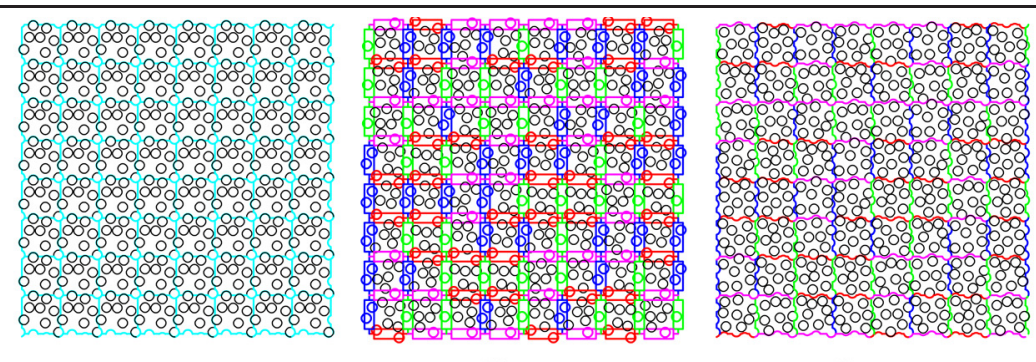

Wang tiling - VW; $\mathbf{n 8}$

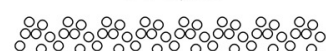

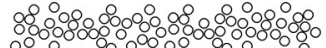

Wang tiling - AW; n8

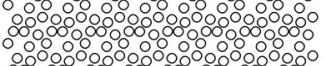

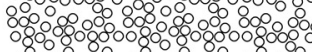

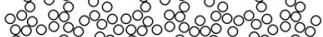

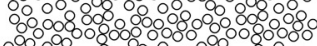

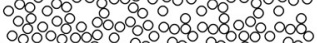

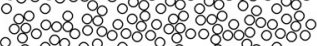

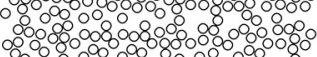

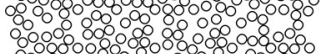

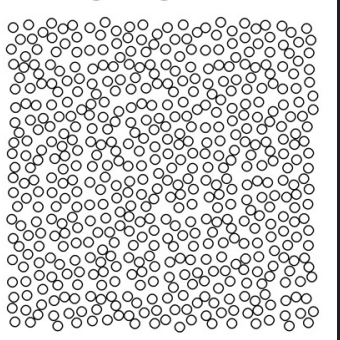

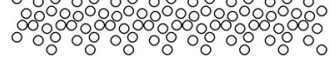
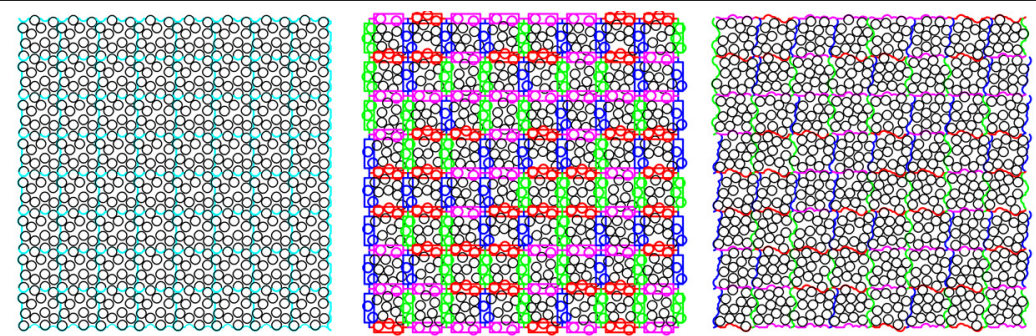

PUC; $\mathbf{n} 12$

Wang tiling - VW; n12

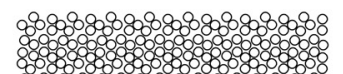

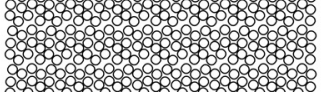

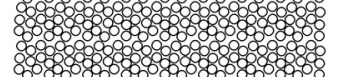

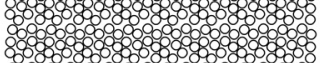

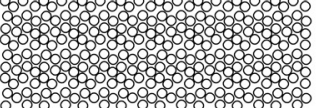

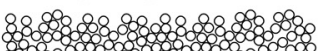

Wang tiling - AW; n12

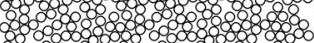

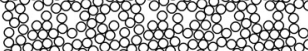

\$8

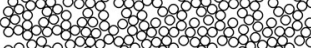

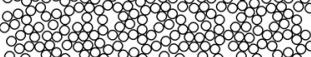

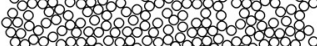

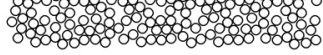

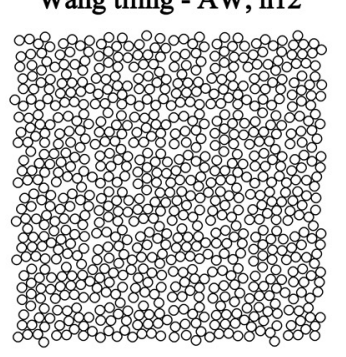

Fig. 7. Generated sets with monodisperse particles - artificial sample, the PUC tiling, the Wang tilings: (upper frame) 4 particles/tile, (middle frame) 8 particles/tile, (lower frame) 12 particles/tile 
The visual evaluation focuses on the artificial periodicity artefacts. The results for each algorithm are briefly commented and encountered problems are pointed out. We are aware of the possible distortion due to the low number of realisations for the visual comparison. Despite this fact, rough insight into the behaviour of the algorithms can be gained and their benefits and disadvantages will be highlighted. The first column of samples represents an initial or original domain with approximately a uniform distribution of particles without significant particle clusters or a regular mesh. It can be seen as a large SEPUC or the RVE.

On the other hand, samples in the second column exhibit clearly visible mesh of repeating areas given by the nature of the PUC concept. This phenomenon is supported by the same size of the tile for all samples of any volume fractions. The PUC for the last case includes twelve particles, whereas for the first one there are only four. Without input changes, representing tile size and number of particles within, the PUC approach cannot compete with the Wang tiling in terms of visual results. Contrary, the lower number of particles in motion leads to savings of computer demands due to the probability of particle collisions. Such assumption prefers the PUC (4 particles needed for the first example) from the VW (26) and the AW (32).

The concept of the Wang tiles with the VW seems promising, especially for examples with a lower volume fraction. Generally, if we want to reduce periodically repeating areas, we have to minimise the number of particles in the border parts of tiles. The best way is to set this number to zero. Such a limit value is, in fact, possible only for cases with very low volume fractions. If a similar consideration was applied to the second analysed example of particle fraction 0.4 , we would have a system with a visible lattice of particle-free regions. Instead, we assigned particles to certain tile parts according to their area ratio. Despite the restriction, dead spaces in the final tiling arise with an increasing sum of particles as a consequence of the algorithm nature.

The last group of tilings with the AW exhibits the greatest visual match with the artificial samples. The visible repetition is primarily given by the number of tiles in the basic set since each tile is completely different. The only exception are the tile corners. If there is a master tile, where any particle finds its position in the corner, the space in all other corners over the whole tile set remains free until another particle fills this area. This result of the game of probabilities is more visible in samples with higher volume fractions. Despite the increasing probability of filling, free corner spaces (if any) cannot hide between other places without particles. Contrary, dilution of artefacts is possible for domains with a lower number of inclusion. Thus, these reconstructions visually better corespond to the random reference medium.

We evaluate the artificial periodicity artefacts also in terms of microstructure statistical description. Special emphasis is placed on secondary peaks in the two-point probability function (S2) plots. The results of this descriptor are based on the probability of finding two points, pixels in our case, within the same phase. The graphic form of generated samples is divided into pixels, where the number of pixels per tile edge equals the size of the tile. Pixels are designated with white colour if a distance from their midpoint to the certain particle centre is lower than the appropriate radius, see Figs. 8, 9, and 10. Therefore, some particles may look incomplete. The principle when each particle has a general template [22] was not implemented here due to the particle motion together with the final random position. The range of statistical description is reduced to $400 \times 400$ points in order to keep results clear and simple. Long range dependencies give no information that would influence the assessment of observed artefacts. The two-point statistics for artificial sample and tiling made of the PUCs corresponds to the binary representation of domains from Fig. 7, shown in the left column, whereas the plotted results for the Wang tiling represent an average of five realisations. The binary representation next to it means one of the realisations and fits the microstructure in Fig. 7. 


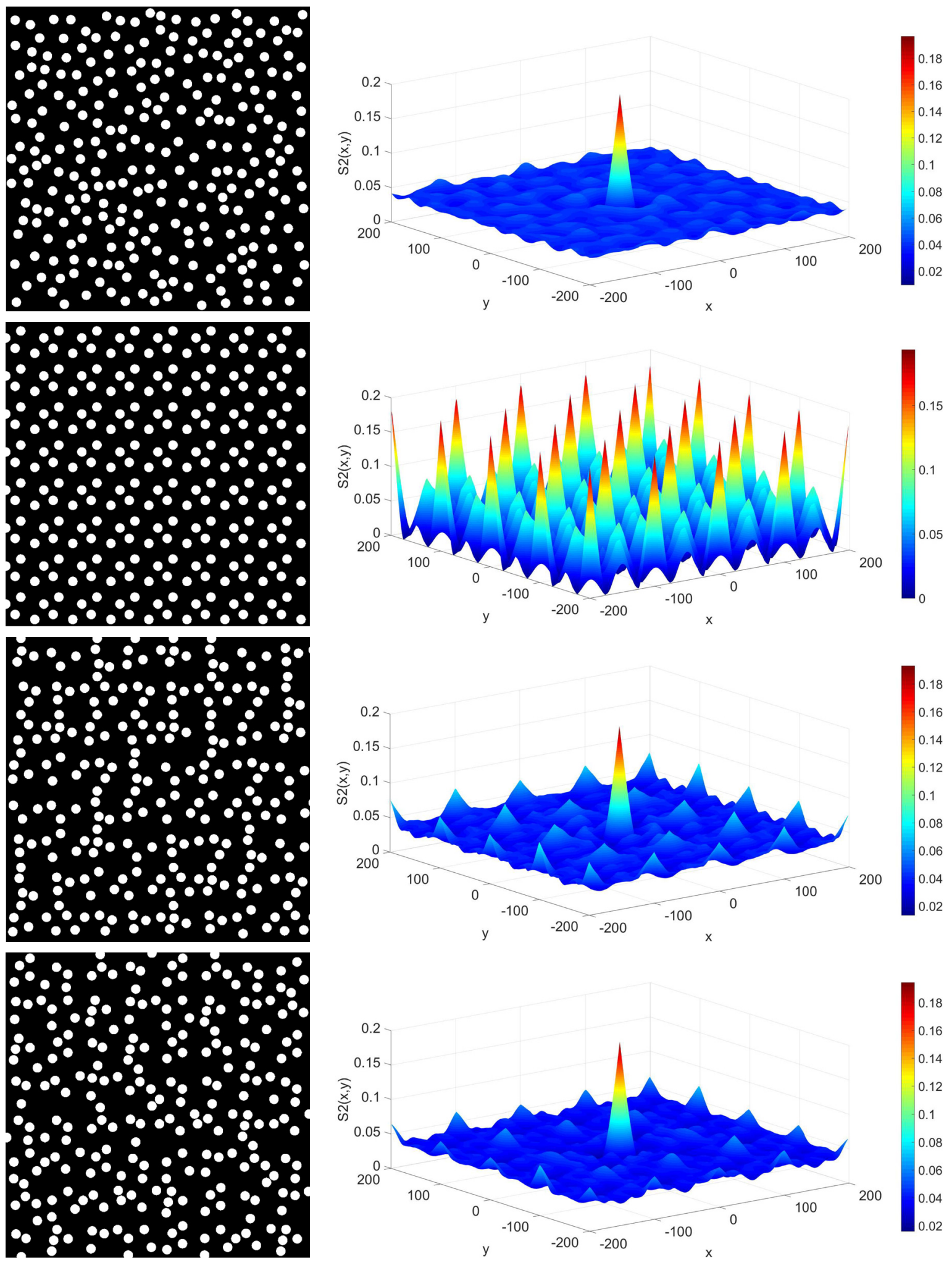

Fig. 8. Analysis of 2D monodisperse samples of volume fraction of approximately 0.2 . Systems from top to bottom: artificial sample, PUC, Wang tiling - VW, Wang tiling - AW; (left) one randomly chosen realisation, (right) S2 function (average of all realisations) 

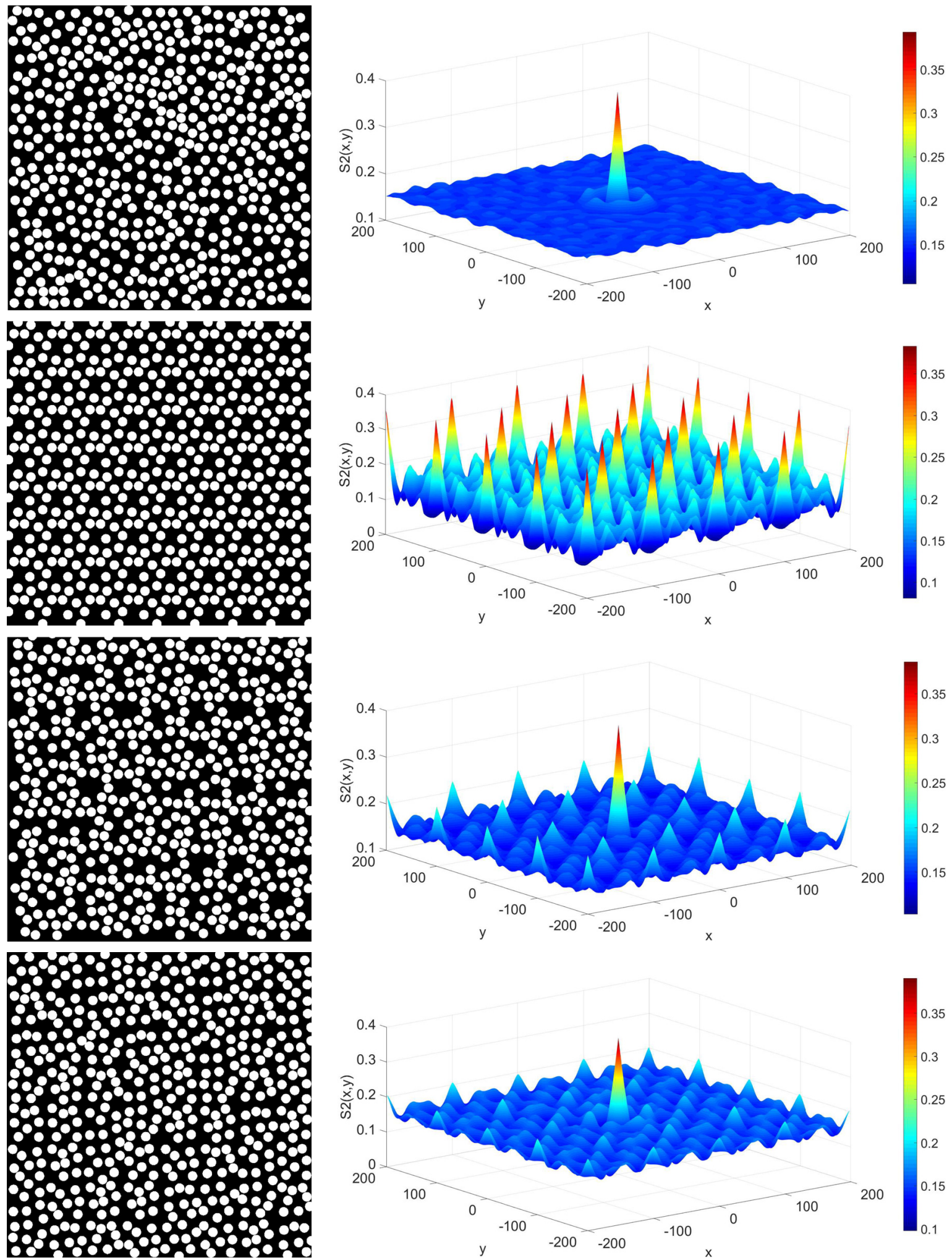

Fig. 9. Analysis of 2D monodisperse samples of the volume fraction of approximately 0.4. Systems from top to bottom: artificial sample, PUC, Wang tiling - VW, Wang tiling - AW; (left) one randomly chosen realisation, (right) S2 function (average of all realisations) 

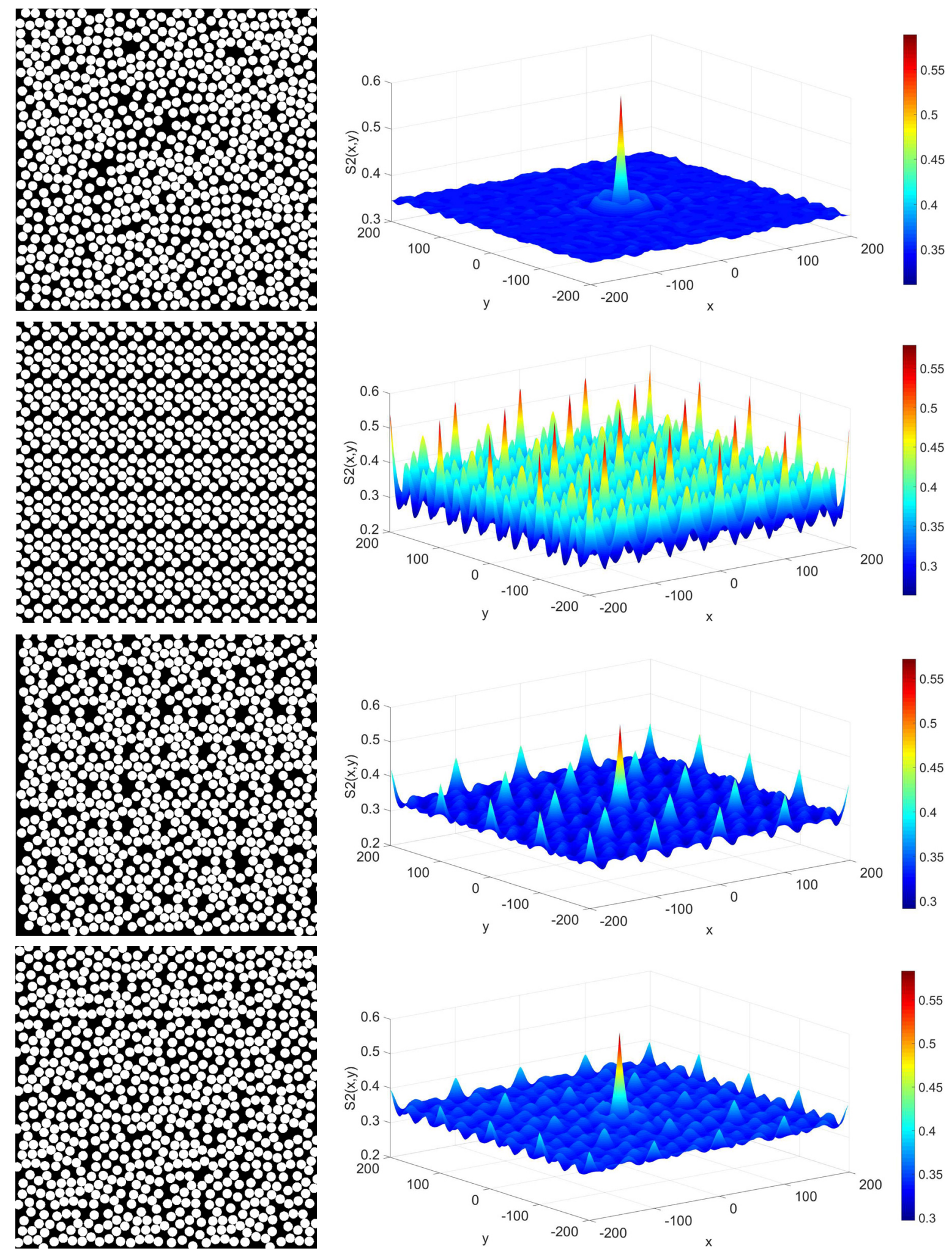

Fig. 10. Analysis of 2D monodisperse samples of the volume fraction of approximately 0.6. Systems from top to bottom: artificial sample, PUC, Wang tiling - VW, Wang tiling - AW; (left) one randomly chosen realisation, (right) S2 function (average of all realisations) 
The discussion of graphical results considers mainly the first and the group of secondary extremes. The highest value in every graph represents the volume fraction of a certain sample in accordance with the description definition. The first extreme is the same for all samples due to the identical total number of particles. However, the prescribed condition of volume fraction is met with an error given by the integer number of particles. Secondary extremes fully reflect the periodicity in the samples. There is no significant secondary peak in the first artificial samples, the situation is different in tiled domains. If we look at the PUC system, every particle in a cell repeats in the main coordinate system in a distance of the tile size with the same probability. Tertiary and other extremes express the geometric relationship between the particles in one cell with the contribution of tile copies in a diagonal direction.

The secondary peaks in domains based on the VW are caused mainly by two phenomena: the regular repetition of boundary particles and the usage of the same tiles. The occurrence probability of both situations is lower than for the PUC system. The first indicated factor does not affect the results of the samples with the AW. An oscillation around the squared volume fraction appears in every tested type of boundary condition as a consequence of the descriptor definition. The comparison in terms of the secondary and tertiary extremes in graphs of statistics is in favour of the AW system.

\subsection{Polydisperse $2 D$ sets}

The algorithms for the generation of the PUC and Wang tiles are capable to work with particles of different radii. Therefore, another group of tested sets represents polydispersible material structures. The overall volume fraction remains the same as for the previous set, but inclusions are of two radii. Their relative volume ratio is approximately 60 per cent for the first radius of 12.5 pixels and 40 per cent for particles of radius 7.5 pixels. The other settings of both material domains and algorithms remain unchanged. The summary of parameters for generated microstructures is presented in Table 2. The visualisation of tilings and appropriate microstructures without tile edges are shown in Fig. 11. The sample labels are supplemented with the number of particles of given radii in each tile.

Table 2. Specification of artificial polydisperse 2D samples

\begin{tabular}{|c|c|c|c|c|c|c|}
\hline \multirow{2}{*}{$\begin{array}{c}\text { Volume } \\
\text { fraction app. }\end{array}$} & \multirow[t]{2}{*}{ Method } & \multicolumn{2}{|c|}{$\begin{array}{l}\text { Particles } \\
\text { per tile }\end{array}$} & \multirow{2}{*}{$\begin{array}{l}\text { Total number } \\
\text { of tiles }\end{array}$} & \multicolumn{2}{|c|}{$\begin{array}{c}\text { Total number } \\
\text { of particles }\end{array}$} \\
\hline & & $r=12.5$ & $r=7.5$ & & $r=12.5$ & $r=7.5$ \\
\hline \multirow{4}{*}{0.2} & sample & - & - & - & 192 & 192 \\
\hline & PUC & 3 & 3 & 64 & 192 & 192 \\
\hline & VW & 4 & 4 & 64 & 192 & 192 \\
\hline & $\overline{\mathrm{AW}}$ & 3 & 3 & 64 & 192 & 192 \\
\hline \multirow{4}{*}{0.4} & sample & - & - & - & 320 & 576 \\
\hline & PUC & 5 & 9 & 64 & 320 & 576 \\
\hline & VW & 7 & 13 & 64 & 320 & 576 \\
\hline & AW & 5 & 9 & 64 & 320 & 576 \\
\hline \multirow{4}{*}{0.6} & sample & - & - & - & 512 & 768 \\
\hline & PUC & 8 & 12 & 64 & 512 & 768 \\
\hline & VW & 11 & 17 & 64 & 512 & 768 \\
\hline & $\mathrm{AW}$ & 8 & 12 & 64 & 512 & 768 \\
\hline
\end{tabular}




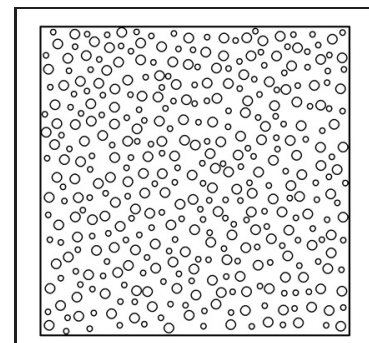

artificial sample

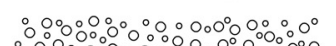

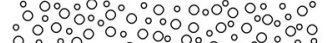

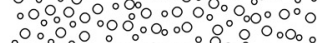

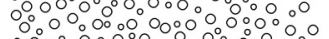

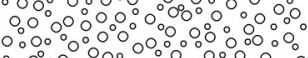

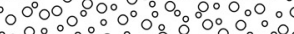

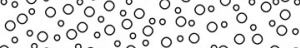

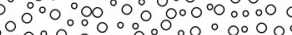

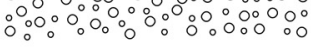

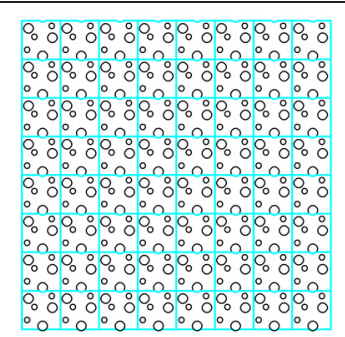

\section{PUC; n3-n3}

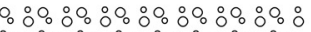

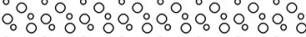

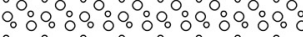
0
$\circ$
$\circ$
$\circ$

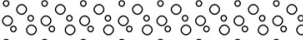

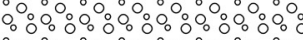

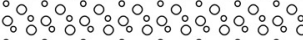

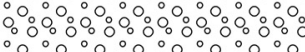

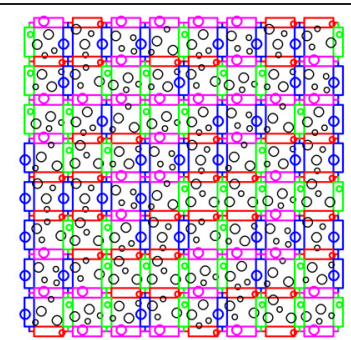

Wang tiling - VW; n3-n3

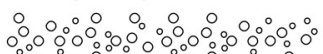
$\therefore 0000000000000000$ 00000000000000

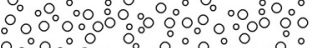
000000000000000000

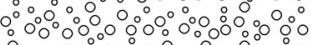
000000000000000000 $\therefore 0000000000000^{\circ} 0^{\circ} \circ 00 \%$

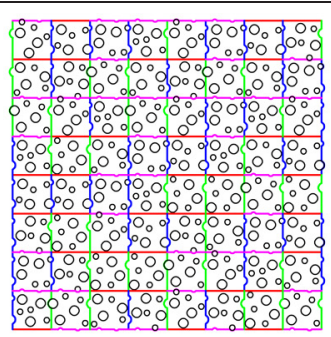

Wang tiling - AW; n3-n3

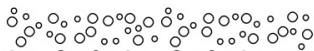
00000000000000000 $\because 00000 \% 0000000$ $\therefore \circ: 00000000000000$ $\therefore 000000000000000$ 000000000000000000

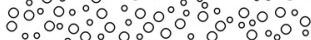
0,00000000000000000000

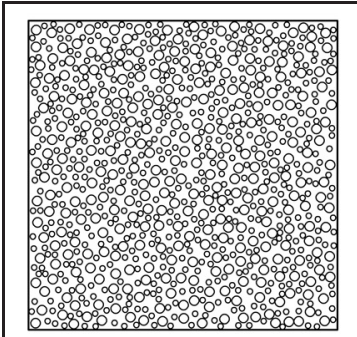

artificial sample

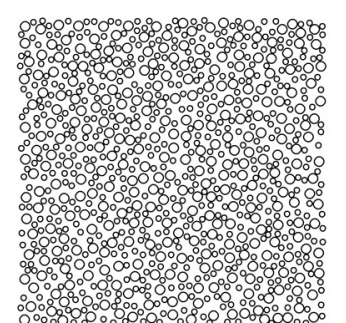

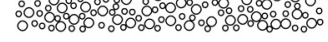

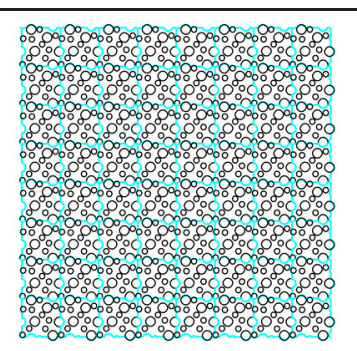

PUC; n5-n9
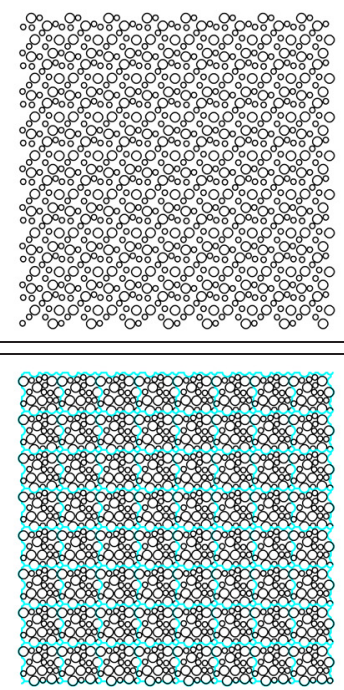

PUC; n8-n12

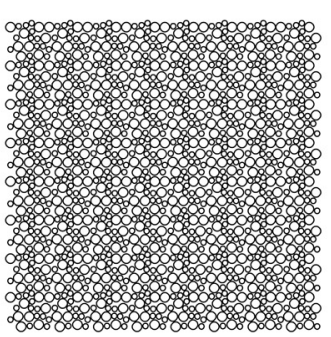

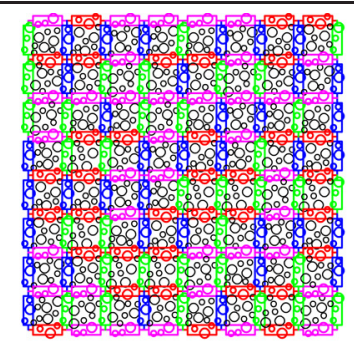

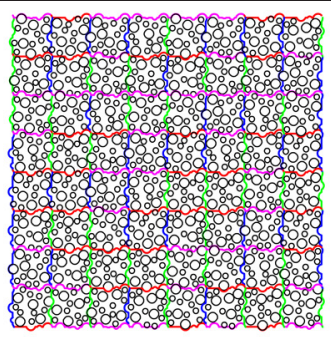

Wang tiling - VW; n5-n9

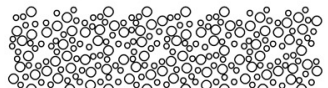

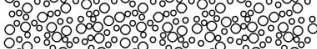

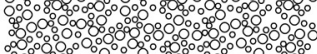

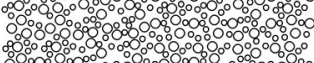

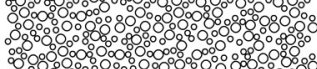

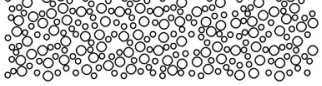

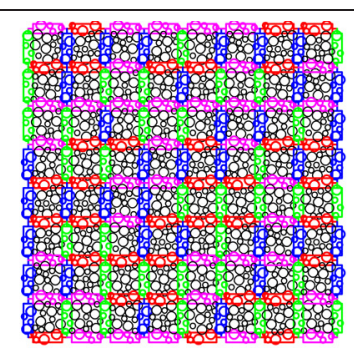

Wang tiling - VW; n8-n12
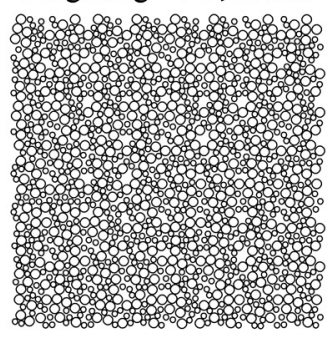

\section{Wang tiling - AW; n5-n9}
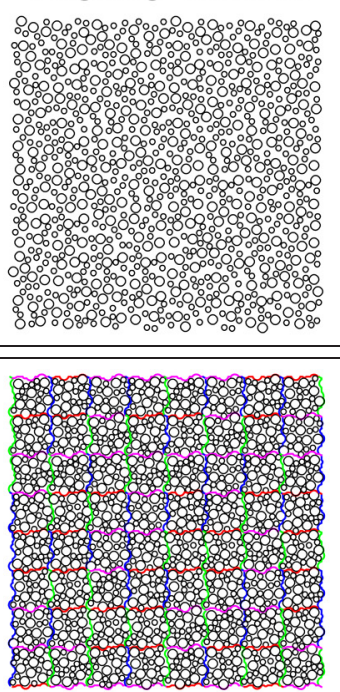

Wang tiling - AW; n8-n12

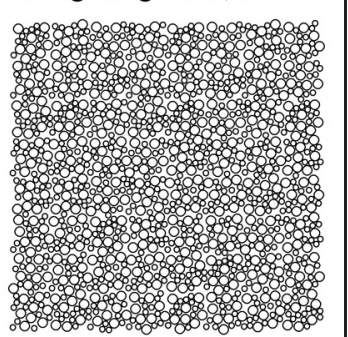

Fig. 11. Generated sets with polydisperse particles - artificial sample, the PUC tiling, the Wang tiling: (upper frame) $3+3$ particles/tile, (middle frame) $5+9$ particles/tile, (lower frame) $8+12$ particles/tile 
The set of polydisperse microstructures for visual comparison includes an artificial sample without tiles and tiled domains of the PUC and Wang tiling with both the VW and AW conditions. The benefits and disadvantages described in the previous sections for monodisperse sets are generally valid in these cases, as well. Emphasis has to be placed on the prescribed volume fraction and the volume ratio of particles of different radii. If we still consider the same tile size, the real volume fraction error gets up to ten per cent for systems with the lowest tile particle number. The lower prescribed volume fraction, the higher the error probability is. This issue can be solved by modifying the tile size according to an integral combination of particles with certain radii. In our study, the tile size is chosen to be the same for the sake of comparison between the proposed methods.

The length of a tile edge or dimensions of border areas are the decisive parameters for the size of dead spaces for the system with the VW. The width of the border areas equals the maximal particle diameter. Thus, if the centre of a small particle reaches the corner, its surface is unable to cover a sufficient free area of the corner dead space. This forms a visually regular mesh of islands without inclusions. Such a phenomenon often appears with a higher volume fraction and a lower ratio of the largest particles to other ones. It can be found in our tested samples, particularly in domains of volume fraction 0.4 and 0.6, see Fig. 13 and Fig. 14, respectively.

On the contrary, polydisperse materials are more suitable for the method with the AW when solving the corner problem. Let us consider a particle of maximal radius, which is located in a corner of the master tile. The deformation occurs in every corner of every tile from the set. One corner in the master tile is occupied with a particle, while all the others represent a free space only. These spaces are filled in by the easiest way with particles of lower radii. Such a particle is "protected" by the master corner inclusion. A collision of the corner master particle with any other particle from the slave tiles occurs earlier than a collision of these particles with the protected one. This advantage of the AW boundary conditions is visible in the modelled states with both monodisperse (Fig. 7) and polydisperse domains (Fig. 11).

Figs. 12-14 complement the visualisation with statistical information. Again, the descriptor is the two-point probability (S2) function. The statistical description of the first two examples of each volume fraction set corresponds to the binary representation located next to a certain graph. The binary form of Wang tiling examples symbolises only one of five realisations, which are used for the statistics. All of these monochrome figures coincide with samples for visual comparison in Fig. 11. If we focus on the secondary extremes of the probability function, we gain a considerable reduction in the Wang tiling case compared to the PUC one. It is caused in particular by two circumstances. The first one is, naturally, usage of different boundary conditions. The second one is the ability to create different tilings via just one set of tiles. Even though the statistical information is averaged for these realisations, each tiling sample itself reduces secondary peaks. Multiple realisations of material domains based on the PUC concept are unnecessary to create and to average the statistics. Considering a statistically homogeneous microstructure, the two-point probability depends only on the relative position of tested pixels. The differences between realisations would only be with regard to the changes in an internal particle arrangement. However, the extremes of the final sample would be the same when there is no change in the tile size. 

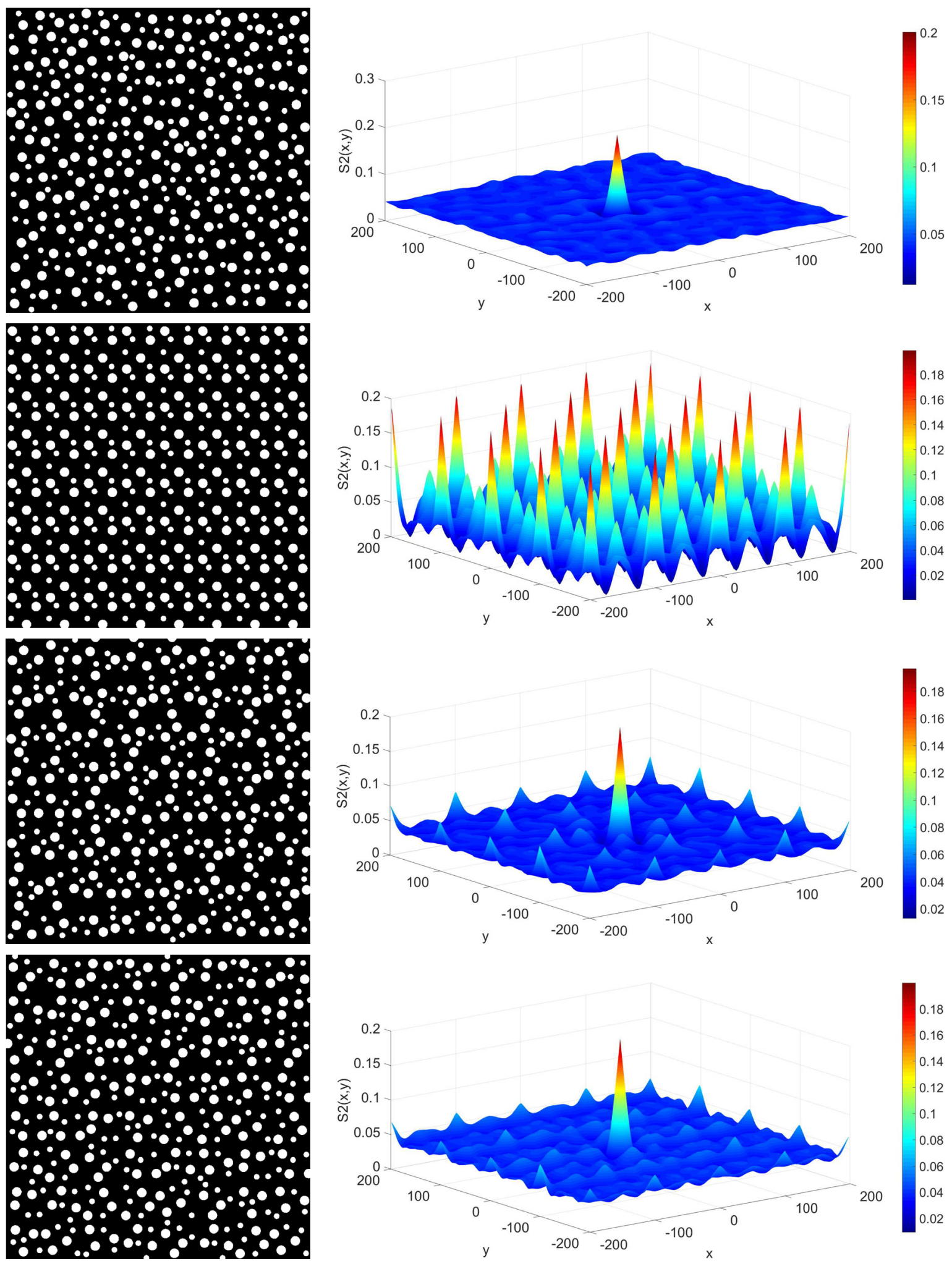

Fig. 12. Analysis of 2D Polydisperse samples of the volume fraction of approximately 0.2. Systems from top to bottom: artificial sample, PUC, Wang tiling - VW, Wang tiling - AW; (left) one randomly chosen realisation, (right) $\mathrm{S} 2$ function (average of all realisations) 

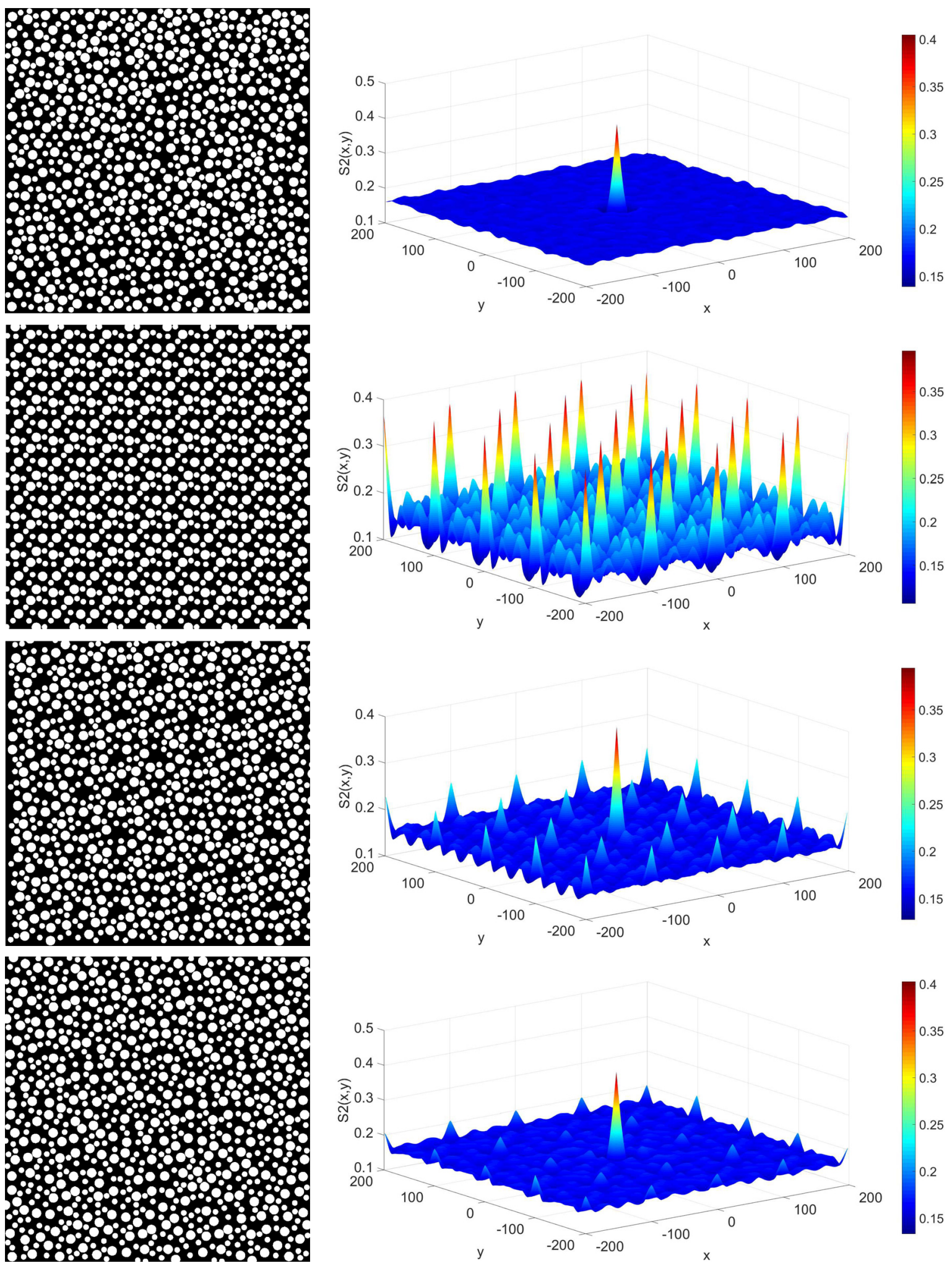

Fig. 13. Analysis of 2D Polydisperse samples of the volume fraction of approximately 0.4 . Systems from top to bottom: artificial sample, PUC, Wang tiling - VW, Wang tiling - AW; (left) one randomly chosen realisation, (right) $\mathrm{S} 2$ function (average of all realisations) 

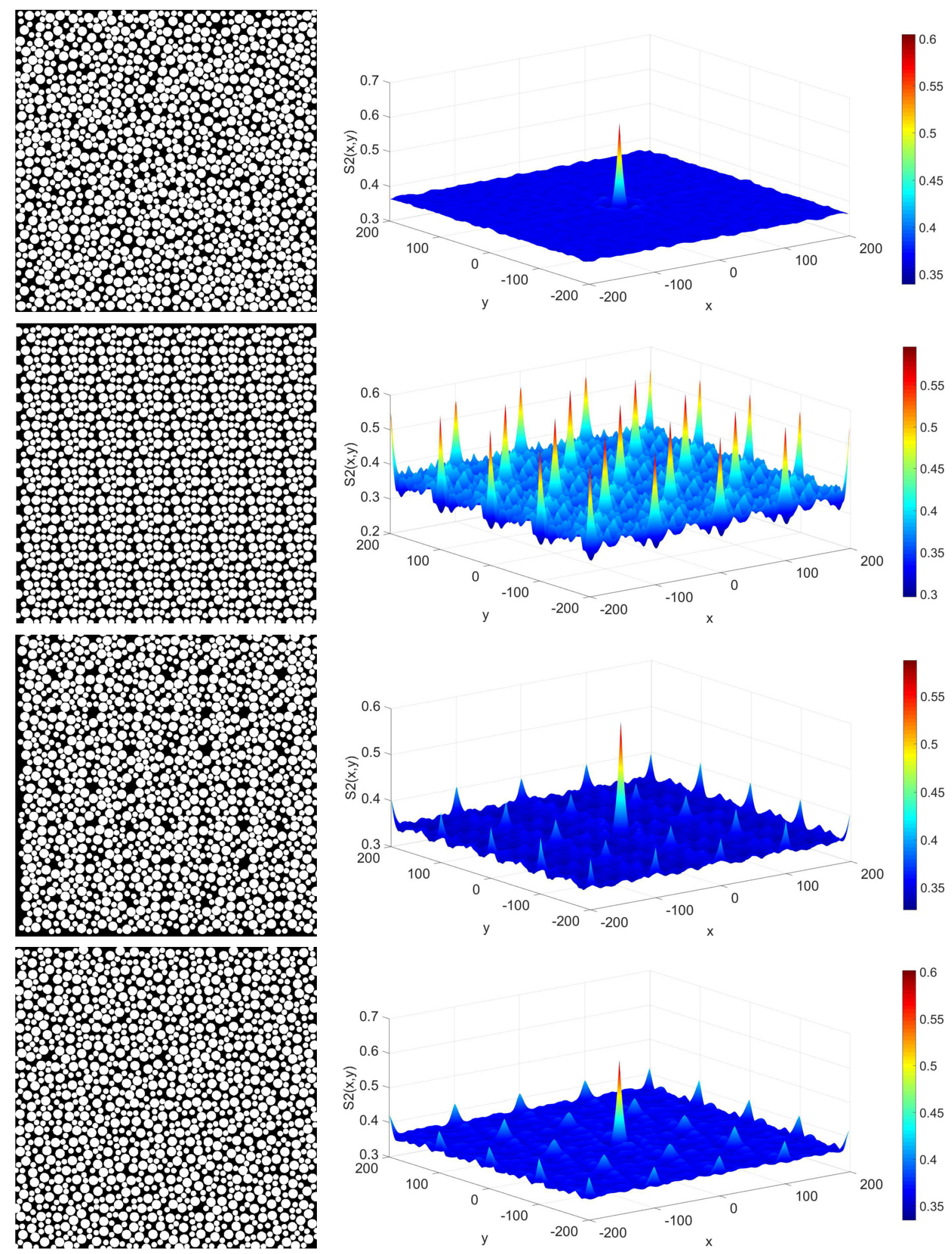

Fig. 14. Analysis of 2D Polydisperse samples of the volume fraction of approximately 0.6. Systems from top to bottom: artificial sample, PUC, Wang tiling - VW, Wang tiling - AW; (left) one randomly chosen realisation, (right) $\mathrm{S} 2$ function (average of all realisations) 


\subsection{Monodisperse $3 D$ sets}

An analysis of real random heterogeneous microstructures in $3 \mathrm{D}$ spaces used to be based on averaging methods and a set of $2 \mathrm{D}$ sections. Nevertheless, complete statistical information or the making of the realistic spatial samples require 3D models. Wang tiling extended into the third dimension allows the creation of such samples. The plane material domains from the previous section are augmented by realisations of the $3 \mathrm{D}$ structure. The samples represent monodisperse impenetrable hard spheres in a continuous phase.

Generated structures are composed of the PUCs and Wang cubes with both the VW and the AW conditions. The comparison of approaches is in terms of unwanted artefacts of artificial periodicity. The form of individual cubes and the particle arrangement are based on principles of modified molecular dynamics as stated in Section 3.1. Thus, the final position of inclusions is randomised, i.e., it depends on initial random velocity vectors. The tested tiling sequence has a shape of cube and consists of 125 individual 3D tiles. Each cube has a size of 40 pixels and contains 12 particles with a radius of 5 pixels. Such a description is valid for PUCs and Wang tiles with the AW boundary condition. Tiles with the VW follow similar rules as for the 2D applications. Duplicated particles in border areas are reduced in the final tiling. However, at the beginning the particles are distributed as follows: one sphere is assigned to every border part and others to the central part. Contrary to the 2D samples, the ratio of the number of particles in border regions to the sum of particles in central parts is lower than the ratio of these tile part volumes. This scheme has been chosen deliberately to demonstrate the benefits of tiling with the VW and to reduce periodicity.

The results of the two-point probability descriptor for 3D applications are hardly visually interpretable. Therefore, a histogram reflecting the number of particles in the spherical shell at a distance $r$ from the certain particle centre is chosen to describe basic statistics, Fig. 15. Similar principles are to be found in radial distribution function descriptor [29]. Concurrently, the last tested spherical shell is in distance equal to the tile dimension, which is sufficient from the point of view of tiling periodicity. The first occupied column denotes sphere particles in distance greater than two particle radii. This is consistent with the geometry since the closest position of two particle centres equals to the sum of their radii. A lower volume fraction together with the randomness of particle location lead to the first column shift for the PUC system. The basic set of Wang tiles includes sixteen individuals, thus the probability of particle placement in the nearest neighbourhood is much higher in comparison to the PUC method.

The observed periodicity phenomenon can be retrieved from the data in the last column as well as from the graph trends. Moreover, the situation, when any column is much higher than its nearest neighbours, indicate the observed phenomenon, as well. The mentioned events can be seen from the statistic of the PUC structure. The last column corresponds to the cube size and includes sphere copies in the main orthogonal directions. The higher values in zone 13, 17, 24 , or 29 contain distances of particles in two closest cubes, but they are significantly affected with copies of particles inside one periodic cell.

The frequency of particles with a relative distance equal to the cube size is significantly lower for Wang tiling with VW in comparison with the PUC concept. The difference is based on the number of particles in the border volume space. In general, a structure of tiling plays also a marginal role. Despite the stochastic tiling process, the final sequence may include neighbours of the same cubes. In our case, such a sequence occurs in less than six per cent of the neighbours. The histogram for Wang tiling with the AW is in agreement with the goal to reduce periodicity artefacts. Here, the frequency in the last column is the lowest from the generated structures. Moreover, the growth rate is fluent indicating uniform distribution without extremes. 

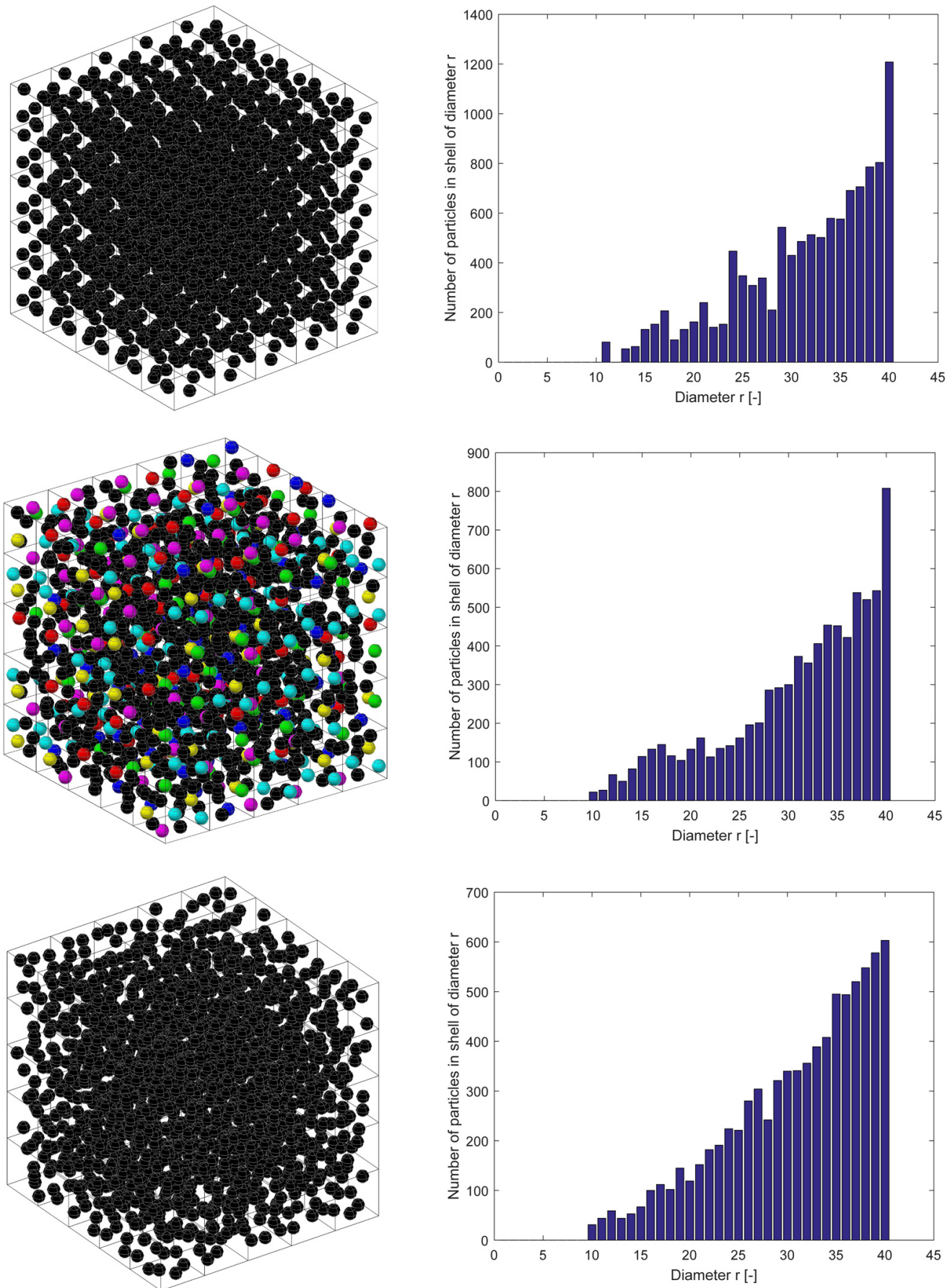

Fig. 15. Analysis of 3D Monodisperse samples of volume fraction approximately 0.08: (left) realisations with the system of PUC, Wang cubes with VW, Wang Cubes with AW, (right) appropriate histograms of particle distances

\section{Conclusions}

This paper dealt with the modelling of random heterogeneous materials composed of hard spheres/circles in a continuous phase. The reconstructed domains were created via both the traditional periodic cell concept and the novel Wang tiling algorithm. Both approaches used principles of molecular dynamics in order to determine the final random particle positions. Special emphasis 
was placed on boundary conditions. Domains with periodic boundary conditions were compared to models based on Wang tiling with i) Volume Walls (VW) and ii) Adaptive Walls (AW).

Generated sets for 2D application included both mono and polydisperse samples, while the 3D models contained particles of the same radii. Analysed examples had three different particle fractions: $0.2,0.4$, and 0.6. The comparison of methods was based on the reduction of unwanted artificial periodicity artefacts. The results of the visual analysis for the $2 \mathrm{D}$ applications pointed to a significant reduction of the observed property with the usage of the AW. This fact was confirmed statistically via the two-point probability descriptor. Similar results were obtained for the 3D microstructures.

Despite a small number of tested samples, Wang tiling with adaptive walls seems to be promising for random heterogeneous material models. It has to be noted that the generated structures have random final particle positions. The task of real microstructure reconstruction requires the Wang tiling to be enriched with optimisation methods. Some contributions can be found, where principles of Simulated Annealing $[4,13]$ were used for the optimisation of Wang tiles [21,22]. However, models with circular particles and molecular dynamics are suitable for utilisation of the Particle Swarm Optimization method [12,23]. These issues together with the implementation of other spherical particles are the main tasks of further work.

\section{Acknowledgements}

The financial support of the Grant Agency of the Czech Technical University in Prague, the grant No.: SGS18/036/OHK1/1T/11, is gratefully acknowledged.

\section{References}

[1] Bargman, S., Klusemann, B., Markmann, J., Schnabel, J.E., Schneider, K., Soyarslan, C., Wilmers, J., Generation of 3D representative volume elements for heterogeneous materials: A review, Progress in Materials Science 96 (2018) 322-384. https://doi.org/10.1016/j.pmatsci.2018.02.003

[2] Berger, R., The undecidability of the domino problem, Memoirs of the American Mathematical Society 66, Providence, 1966. https://doi.org/10.1090/memo/0066

[3] Cohen, M.F., Shade, J., Hiller, S., Deussen, O., Wang tiles for image and texture generation, Proceedings of the conference SIGGRAPH '03, New York, ACM Press, 2003, pp. 287-294. https://doi.org/10.1145/1201775.882265

[4] Černý, V., Thermodynamical approach to the traveling salesman problem: An efficient simulation algorithm, Journal of Optimization Theory and Applications 45 (1) (1985) 41-51. https://doi.org/10.1007/BF00940812

[5] Čulík, K., An aperiodic set of 13 Wang tiles, Discrete Mathematics 160 (1-3) (1996) 245-251. https://doi.org/10.1016/S0012-365X(96)00118-5

[6] Donev, A., Jammed packings of hard particles, Ph.D. thesis, Princeton University, Princeton, 2006.

[7] Doškár̆, M., Novák, J., Zeman, J., Aperiodic compression and reconstruction of real-world material systems based on Wang tiles, Physical Review E 90 (6) (2014) No. 062118. https://doi.org/10.1103/PhysRevE.90.062118

[8] Fullwood, D. T., Niezgoda, S.R., Adams, B.L., Kalidindi, S. R., Microstructure sensitive design for performance optimization, Progress in Materials Science 55 (6) (2010) 477-562. https://doi.org/10.1016/j.pmatsci.2009.08.002

[9] Hifi, M., M'Hallah, R., A literature review on circle and sphere packing problems: Models and methodologies, Advances in Operations Research 2009 (2009) 1-22.

https://doi.org/10.1155/2009/150624 


\section{D. Šedlbauer et al. / Applied and Computational Mechanics 13 (2019) 53-76}

[10] Hill, R., Elastic properties of reinforced solids: Some theoretical principles, Journal of the Mechanics and Physics of Solids 11 (5) (1963) 357-372. https://doi.org/10.1016/0022-5096(63)90036-X

[11] Jeandel, E., Rao, M., An aperiodic set of 11 Wang tiles, Report No. al-01166053v2, Inria, 2015.

[12] Kennedy, J., Eberhart, R. C., Particle swarm optimization, Proceedings of the 1995 IEEE international conference on neural networks, Perth, 1995, pp. 1942-1948.

[13] Kirkpatrick, S., Gelatt, C.D., Vecchi, M.P., Optimization by simulated annealing, Science 220 (4598) (1983) 671-680. https://doi.org/10.1126/science.220.4598.671

[14] Klein, T., Autin, L., Kozlíková, B., Goodsell, D. S., Olson, A., Groller, M. E., Viola, I., Instant construction and visualization of crowded biological environments, IEEE Transactions on Visualization and Computer Graphics 24 (1) (2018) 862-872. https://doi.org/10.1109/TVCG.2017.2744258

[15] Kumar, N.C., Matouš, K., Geubelle, P.H., Reconstruction of periodic unit cells of multimodal random particulate composites using genetic algorithms, Computational Materials Science 42 (2) (2008) 352-367. https://doi.org/10.1016/j.commatsci.2007.07.043

[16] Lagae, A., Tile-based methods in computer graphics, Ph.D. thesis, Catholic University of Leuven, Leuven, 2007.

[17] Lagae, A., Dutré, P., An alternative for Wang tiles, ACM Transactions on Graphics 25 (4) (2006) 1442-1459. https://doi.org/10.1145/1183287.1183296

[18] Lubachevsky, B.D., Stillinger, F. H., Geometric properties of random disk packings, Journal of Statistical Physics 60 (5-6) (1990) 561-583. https://doi.org/10.1007/BF01025983

[19] Lubachevsky, B.D., Stillinger, F. H., Pinson, E. N., Disks vs. spheres: Contrasting properties of random packings, Journal of Statistical Physics 64 (3-4) (1991) 501-524. https://doi.org/10.1007/BF01048304

[20] Moussaddy, H., Therriault, D., Lévesque, M., Assessment of existing and introduction of a new and robust efficient definition of the representative volume element, International Journal of Solids and Structures 50 (24) (2013) 3817-3828. https://doi.org/10.1016/j.ijsolstr.2013.07.016

[21] Novák, J., Kučerová, A., Zeman, J., Compressing random microstructures via stochastic Wang tilings, Physical Review E 86 (4) (2012) No. 040104. https://doi.org/10.1103/PhysRevE.86.040104

[22] Novák, J., Kučerová, A., Zeman, J., Microstructural enrichment functions based on stochastic Wang tilings, Modelling and Simulation in Materials Science and Engineering 21 (2) (2013) No. 025014. https://doi.org/10.1088/0965-0393/21/2/025014

[23] Reynolds, C. W., Flocks, herds and schools: A distributed behavioral model, ACM SIGGRAPH Computer Graphics 21 (4) (1987) 25-34. https://doi.org/10.1145/37402.37406

[24] Salmi, M., Auslender, F., Bornert, M., Fogli, M., Various estimates of representative volume element sizes based on a statistical analysis of the apparent behavior of random linear composites, Comptes Rendus Mécanique 340 (4-5) (2012) 230-246.

https://doi.org/10.1016/j.crme.2012.02.007

[25] Seeman, N.C., Sleiman, H.F., DNA nanotechnology, Nature Reviews Materials 3 (1) (2017) No. 17068. https://doi.org/10.1038/natrevmats.2017.68

[26] Šedlbauer, D., Adaptive boundaries of Wang tiles for heterogeneous material modelling, Advanced Materials Research 1144 (2017) 159-166. https://doi.org/10.4028/www.scientific.net/AMR.1144.159

[27] Šedlbauer, D., Heterogeneous material modelling via dynamics packing of stochastic Wang tiles, Proceedings of the 4th Conference Nano \& Macro Mechanics, Czech Technical University in Prague, Prague, 2013, pp. 187-192.

[28] Šejnoha, M., Zeman, J., Micromechanics in practice, WIT Press, Ashurst, 2013.

[29] Torquato, S., Random heterogeneous materials: Microstructure and macroscopic properties, New York: Springer, 2002. https://doi.org/10.1115/1.1483342 
D. Šedlbauer et al. / Applied and Computational Mechanics 13 (2019) 53-76

[30] Wang, H., Proving theorems by pattern recognition I., Communications of the ACM 3 (4) (1960) 220-234. https://doi.org/10.1145/367177.367224

[31] Wang, H., Proving theorems by pattern recognition II., Bell System Technical Journal 40 (1) (1961) 1-41. https://doi.org/10.1002/j.1538-7305.1961.tb03975.x

[32] Winfree, E., Liu, F., Wenzler, L. A., Seeman, N. C., Design and self-assembly of two-dimensional DNA crystals, Nature 394 (6693) (1998) 539-544. https://doi.org/10.1038/28998 